\title{
Connected, Bounded Degree, Triangle Avoidance Games
}

\author{
Nishali Mehta* \\ The Ohio State University \\ nishali@math.ohio-state.edu \\ Ákos Seress* \\ The Ohio State University \\ Centre for Mathematics of Symmetry and Computation \\ University of Western Australia \\ akos@math.ohio-state.edu \\ Submitted: May 31, 2010; Accepted: Sept 6, 2011; Published: Sep 26, 2011 \\ Mathematics Subject Classification: 05C57

\begin{abstract}
We consider variants of the triangle-avoidance game first defined by Harary and rediscovered by Hajnal a few years later. A graph game begins with two players and an empty graph on $n$ vertices. The two players take turns choosing edges within $K_{n}$, building up a simple graph. The edges must be chosen according to a set of restrictions $\mathcal{R}$. The winner is the last player to choose an edge that does not violate any of the restrictions in $\mathcal{R}$. For fixed $n$ and $\mathcal{R}$, one of the players has a winning strategy. For a pair of games where $\mathcal{R}$ includes bounded degree, connectedness, and triangle-avoidance, we determine the winner for all values of $n$.
\end{abstract}

\section{Introduction}

Two players, $\mathcal{A}$ and $\mathcal{B}$, begin with an empty graph on $n$ vertices, where $n \geq 3$. Player $\mathcal{A}$ goes first, choosing an edge between two vertices. The two players take turns choosing edges within $K_{n}$, building up a simple graph. The edges have to be chosen according to a set of restrictions $\mathcal{R}$. The winner is the last player to choose an edge that does not violate any of the restrictions in $\mathcal{R}$. For fixed $n$ and $\mathcal{R}$, one of the players has a winning strategy. Let $\Gamma_{\mathcal{R}}(n)$ represent the game with restriction set $\mathcal{R}$ on $n$ vertices. Let $f_{\mathcal{R}}: \mathbb{N} \backslash\{1,2\} \rightarrow\{\mathcal{A}, \mathcal{B}\}$ be the function defined by

\footnotetext{
${ }^{*}$ Research partially supported by the NSF and by ARC Grant DP1096525.
} 


$$
f_{\mathcal{R}}(n)=\text { winner of } \Gamma_{\mathcal{R}}(n)
$$

We will look at games with one or more of the following restrictions and determine the winner for all values of $n$.

Let $G(V, E) \leq K_{n}$ be the graph made up of all edges chosen so far in the game. Let $u, v \in V, u \neq v,(u, v) \notin E$.

Restriction B2 (Bounded Degree). If $\max \left\{\operatorname{deg}_{G}(u), \operatorname{deg} g_{G}(v)\right\} \geq 2$, then the edge $(u, v)$ cannot be chosen.

Restriction B3 (Bounded Degree). If $\max \left\{\operatorname{deg}_{G}(u), \operatorname{deg}_{G}(v)\right\} \geq 3$, then the edge $(u, v)$ cannot be chosen.

Restriction $\mathbf{T}$ (Triangle Avoidance). If $\exists w \in V \backslash\{u, v\}$ such that both edges $(u, w),(v, w) \in E$, then the edge $(u, v)$ cannot be chosen.

Let $M$ be the upper bound on the vertex degree, e.g. $M=3$ if $\mathcal{R}$ includes Restriction $B 3$. When no upper bound is specified, $M=n-1$.

Restriction $\mathbf{C}$ (Connectedness). If $\operatorname{deg}_{G}(u)=0, \operatorname{deg}_{G}(v)=0$ and there is $w \in V$ such that $0<\operatorname{deg}_{G}(w)<M$, then the edge $(u, v)$ cannot be chosen.

In this paper we prove the following two theorems:

Theorem 4.5. For $n \geq 5, f_{\{B 3, C\}}(n)=\mathcal{B} \Longleftrightarrow n \equiv 2(\bmod 4)$.

Theorem 5.6. For $n \geq 12, f_{\{B 3, T, C\}}(n)=\mathcal{B} \Longleftrightarrow n \equiv 1,2(\bmod 4)$.

The games with $\mathcal{R}=\{B 2, C\}$ and $\mathcal{R}=\{B 2, T, C\}$ are relatively simple. As a result, it is not too difficult to show the following.

Proposition. For $n \geq 4, f_{\{B 2, C\}}(n)=\mathcal{A} \Longleftrightarrow n \equiv 0(\bmod 2)$. For $n \geq 3, f_{\{B 2, T, C\}}(n)=$ $\mathcal{A} \Longleftrightarrow n \equiv 2(\bmod 4)$.

In an accompanying paper [5], we give a complete analysis of the four games with $\mathcal{R} \subset\{B 2, B 3, T\}$ and $|\mathcal{R} \cap\{B 2, B 3\}|=1$.

\section{Background}

The triangle avoidance game $\Gamma_{\{T\}}(n)$, suggested by Frank Harary [3] and six years later by András Hajnal, remains open in the general case. The function $f_{\{T\}}(n)$ is known for values of $n \leq 15$. The winners for $n \leq 12$ were computed by Cater, Harary, and Robinson [1]. Pralat [6] computed the winners for $n=13,14,15$. Gordinowicz and Pralat [2] computed the winner for $n=16$.

\begin{tabular}{c|c|c|c|c|c|c|c|c|c|c|c|c|c|c}
$n$ & 3 & 4 & 5 & 6 & 7 & 8 & 9 & 10 & 11 & 12 & 13 & 14 & 15 & 16 \\
\hline$f_{\{T\}}(n)$ & $\mathcal{B}$ & $\mathcal{B}$ & $\mathcal{B}$ & $\mathcal{A}$ & $\mathcal{B}$ & $\mathcal{B}$ & $\mathcal{B}$ & $\mathcal{A}$ & $\mathcal{B}$ & $\mathcal{A}$ & $\mathcal{A}$ & $\mathcal{A}$ & $\mathcal{A}$ & $\mathcal{A}$
\end{tabular}


The connected version of the triangle avoidance game, $\Gamma_{\{T, C\}}(n)$, was solved by Seress [7]. He proved $f_{\{T, C\}}(n)=\mathcal{A} \Longleftrightarrow n \equiv 0(\bmod 2)$.

This paper and the accompanying paper by Mehta and Seress [5], are based on the thesis work by Mehta [4].

\section{Definitions}

When $|V|=n$, write $V=\left\{v_{1}, \ldots, v_{n}\right\}$. Let $e_{i}$ denote the $i$ th edge chosen and $E_{k}=$ $\left\{e_{1}, \ldots, e_{k}\right\}$. For given $n$ and $k$, let $G_{n, k}$ be the graph on vertex set $V$ with edge set $E_{k}$. The graphs $G=G_{n, k}$ we consider will always satisfy $\operatorname{deg}_{G}(v) \leq 3$ for all vertices $v \in V$.

We make the following convention, simplifying the description of the games. The ordering of $V$ defines a natural lexicographic ordering < of the edges of the complete graph $K_{n}$. Given a graph $G_{n, k}$, if the next player chooses the edge $e_{k+1}$ then there is no edge $e<e_{k+1}$ such that $G_{n, k+1} \simeq G_{n, k} \cup e$.

A vertex $v_{i}$ is said to be out of play if, $\forall v_{j} \in V \backslash\left\{v_{i}\right\}$ such that $\left(v_{i}, v_{j}\right) \notin E$, choosing the edge $\left(v_{i}, v_{j}\right)$ will violate a restriction in $\mathcal{R}$. A vertex $v_{i}$ that is not out of play is in play. In each game mentioned here, once a vertex is out of play it remains out of play for the rest of the game.

We make a second convention, further simplifying the description. Given a graph $G_{n, k}$, if the next player chooses the edge $e_{k+1}$ then there is no edge $e<e_{k+1}$ such that game play for the remainder of the game is identical in $G_{n, k+1}$ and $G_{n, k} \cup e$. (This situation can occur if the induced subgraphs on the vertices still in play are isomorphic, even if the graphs themselves are not.)

The choice of the $k$ th edge $e_{k}$ will be called the kth round.

Define sets $Z, P, T \subset V$ on a graph $G$ according to vertex degree:

$$
\begin{aligned}
& Z(G)=\left\{v \in V(G): \operatorname{deg}_{G}(v)=0\right\}, \quad z(G)=|Z(G)| \\
& P(G)=\left\{v \in V(G): \operatorname{deg}_{G}(v)=1\right\}, \quad p(G)=|P(G)| \\
& T(G)=\left\{v \in V(G): \operatorname{deg}_{G}(v)=2\right\}, \quad t(G)=|T(G)| .
\end{aligned}
$$

\section{$4 \mathcal{R}=\{B 3, C\}$}

In the game $\Gamma_{\{B 3, C\}}(n)$, we define graph classes $\mathcal{K}_{i}(z)$ for $i=1,2,3,4$, where $z \geq 0$ is an integer. One player can maintain that, for particular values of $k$, the graph $G_{n, k} \in \mathcal{K}_{i}(z)$ for some $i$. That is, this player does not stay within $\mathcal{K}_{i}(z)$ after each of his turns, but regularly returns to it after a few rounds. Following this strategy until the end of the game forces a win.

Define sets of graphs $\mathcal{K}_{i}(z)$ for $i=1,2,3,4$ as follows:

1. For any integer $z \geq 0, G(V, E) \in \mathcal{K}_{1}(z) \Longleftrightarrow z(G)=z, p(G)=0$, and $t(G)=2$, with $T(G)=\left\{u_{1}, u_{2}\right\},\left(u_{1}, u_{2}\right) \in E$.

2. For any integer $z \geq 0, G(V, E) \in \mathcal{K}_{2}(z) \Longleftrightarrow z(G)=z, p(G)=0$, and $t(G)=1$. 
3. For any integer $z \geq 0, G(V, E) \in \mathcal{K}_{3}(z) \Longleftrightarrow z(G)=z, p(G)=1$, and $t(G)=0$.

4. For any integer $z \geq 0, G(V, E) \in \mathcal{K}_{4}(z) \Longleftrightarrow z(G)=z, p(G)=1$, and $t(G)=1$, with $u_{1} \in P(G), u_{2} \in T(G),\left(u_{1}, u_{2}\right) \in E$.

Lemma 4.1. For $n \geq 3$, in $\Gamma_{\{B 3, C\}}(n)$ if either player chooses an edge that creates a graph in $\mathcal{K}_{1}(z)$ with $z \equiv 0(\bmod 4)$ then that player has a winning strategy.

Proof. Let $n \geq 3$. Suppose the edges $e_{1}, \ldots, e_{k-1}$ have already been chosen. Suppose player $\mathcal{A}$ chooses the $k$ th edge so that $G_{n, k} \in \mathcal{K}_{1}(z)$ with $z \equiv 0(\bmod 4)$. Then $T\left(G_{n, k}\right)=$ $\left\{u_{1}, u_{2}\right\}$ with $\left(u_{1}, u_{2}\right) \in E_{k}$. The strategy described here will work for $\mathcal{B}$ as well. We proceed by induction on $z$.

Base case: $z=0$. All vertices are out of play except $u_{1}$ and $u_{2}$, which are already connected by an edge. Since no new edge can be chosen, $\mathcal{A}$ wins.

Assume for induction that the statement holds when $z=4 m$ for some $m \geq 0$. Suppose $z=4(m+1)$. Let $Z\left(G_{n, k}\right)=\left\{w_{1}, \ldots, w_{4 m+4}\right\}$. Each vertex $v \notin\left(Z\left(G_{n, k}\right) \cup T\left(G_{n, k}\right)\right)$ has $\operatorname{deg}_{G_{n, k}}(v)=3$ and is out of play.

Up to game play equivalence, $\mathcal{B}$ must choose $\left(u_{1}, w_{1}\right)$. $\mathcal{A}$ chooses $\left(u_{2}, w_{1}\right)$. $\mathcal{B}$ must choose $\left(w_{1}, w_{2}\right)$. $\mathcal{A}$ chooses $\left(w_{2}, w_{3}\right)$. $\mathcal{B}$ has two choices: $\left(w_{2}, w_{4}\right)$ or $\left(w_{3}, w_{4}\right)$. $\mathcal{A}$ chooses whichever edge $\mathcal{B}$ did not. Now vertices $u_{1}, u_{2}, w_{1}, w_{2}$ are out of play, $z\left(G_{n, k+6}\right)=4 m$, $p\left(G_{n, k+6}\right)=0$, and $t\left(G_{n, k+6}\right)=2$ with $T\left(G_{n, k+6}\right)=\left\{w_{3}, w_{4}\right\},\left(w_{3}, w_{4}\right) \in E_{k+6}$. Thus $G_{n, k+6} \in \mathcal{K}_{1}(4 m)$, so $\mathcal{A}$ wins by induction.

Lemma 4.2. For $n \geq 3$, in $\Gamma_{\{B 3, C\}}(n)$ if either player chooses an edge that creates a graph in $\mathcal{K}_{2}(z)$ with $z \equiv 0(\bmod 4)$ then that player has a winning strategy.

Proof. Let $n \geq 3$. Suppose the edges $e_{1}, \ldots, e_{k-1}$ have already been chosen. Suppose player $\mathcal{A}$ chooses the $k$ th edge so that $G_{n, k} \in \mathcal{K}_{2}(z)$ with $z \equiv 0(\bmod 4)$. The strategy described here will work for $\mathcal{B}$ as well. We proceed by induction on $z$.

Base case: $z=0$. All but one vertex are out of play, so no new edge can be chosen. Thus $\mathcal{A}$ wins.

Assume for induction that the statement holds when $z=4 m$ for some $m \geq 0$. Suppose $z=4(m+1)$. Let $Z\left(G_{n, k}\right)=\left\{w_{1}, \ldots, w_{4 m+4}\right\}$. Let $T\left(G_{n, k}\right)=\{u\}$. Each vertex $v \notin$ $\left(Z\left(G_{n, k}\right) \cup T\left(G_{n, k}\right)\right)$ has $\operatorname{deg}_{G_{n, k}}(v)=3$ and is out of play.

Up to isomorphism, $\mathcal{B}$ must choose $\left(u, w_{1}\right)$. $\mathcal{A}$ chooses $\left(w_{1}, w_{2}\right) . \mathcal{B}$ has two choices: $\left(w_{1}, w_{3}\right)$ or $\left(w_{2}, w_{3}\right)$. $\mathcal{A}$ chooses whichever edge $\mathcal{B}$ did not. $\mathcal{B}$ must choose $\left(w_{2}, w_{4}\right)$. $\mathcal{A}$ chooses $\left(w_{3}, w_{4}\right)$. Now vertices $u, w_{1}, w_{2}, w_{3}$ are out of play, $z\left(G_{n, k+6}\right)=4 m, p\left(G_{n, k+6}\right)=$ 0 , and $t\left(G_{n, k+6}\right)=1$. Thus $G_{n, k+6} \in \mathcal{K}_{2}(4 m)$, so $\mathcal{A}$ wins by induction.

Lemma 4.3. For $n \geq 3$, in $\Gamma_{\{B 3, C\}}(n)$ if either player chooses an edge that creates a graph in $\mathcal{K}_{3}(z)$ with $z \equiv 0(\bmod 4)$ then that player has a winning strategy.

Proof. Let $n \geq 3$. Suppose the edges $e_{1}, \ldots, e_{k-1}$ have already been chosen. Suppose player $\mathcal{A}$ chooses the $k$ th edge so that $G_{n, k} \in \mathcal{K}_{3}(z)$ with $z \equiv 0(\bmod 4)$. The strategy described here will work for $\mathcal{B}$ as well. We proceed by induction. 
Base case: $z=0$. All but one vertex are out of play, so no new edge can be chosen. Thus $\mathcal{A}$ wins.

Assume for induction that the statement holds when $z=4 m$ for some $m \geq 0$. Suppose $z=4(m+1)$. Let $Z\left(G_{n, k}\right)=\left\{w_{1}, \ldots, w_{4 m+4}\right\}$. Let $P\left(G_{n, k}\right)=\{u\}$. Each vertex $v \notin\left(Z\left(G_{n, k}\right) \cup P\left(G_{n, k}\right)\right)$ has $\operatorname{deg}_{G_{n, k}}(v)=3$ and is out of play.

Up to isomorphism, $\mathcal{B}$ must choose $\left(u, w_{1}\right)$. $\mathcal{A}$ chooses $\left(u, w_{2}\right) . \mathcal{B}$ has two choices: $\left(w_{1}, w_{3}\right)$ or $\left(w_{1}, w_{2}\right)$. $\mathcal{A}$ chooses whichever edge $\mathcal{B}$ did not. $\mathcal{B}$ has three choices: $\left(w_{2}, w_{3}\right)$, $\left(w_{2}, w_{4}\right)$, or $\left(w_{3}, w_{4}\right)$.

- If $\mathcal{B}$ chooses $\left(w_{2}, w_{3}\right)$ then $\mathcal{A}$ chooses $\left(w_{3}, w_{4}\right)$. Now vertices $u, w_{1}, w_{2}, w_{3}$ are out of play, $z\left(G_{n, k+6}\right)=4 m, p\left(G_{n, k+6}\right)=1$, and $t\left(G_{n, k+6}\right)=0$. Thus $G_{n, k+6} \in \mathcal{K}_{3}(4 m)$, so $\mathcal{A}$ wins by induction.

- If $\mathcal{B}$ chooses one of $\left(w_{2}, w_{4}\right)$ or $\left(w_{3}, w_{4}\right)$, then $\mathcal{A}$ chooses the other edge. Now vertices $u, w_{1}, w_{2}$ are out of play, $z\left(G_{n, k+6}\right)=4 m, p\left(G_{n, k+6}\right)=0$, and $t\left(G_{n, k+6}\right)=2$ with $T\left(G_{n, k+6}\right)=\left\{w_{3}, w_{4}\right\},\left(w_{3}, w_{4}\right) \in E_{k+6}$. Thus $G_{n, k+6} \in \mathcal{K}_{1}(4 m)$, so $\mathcal{A}$ wins by Lemma 4.1.

Lemma 4.4. For $n \geq 3$, in $\Gamma_{\{B 3, C\}}(n)$ if either player chooses an edge that creates a graph in $\mathcal{K}_{4}(z)$ with $z \equiv 0(\bmod 4)$ then that player has a winning strategy.

Proof. Let $n \geq 3$. Suppose the edges $e_{1}, \ldots, e_{k-1}$ have already been chosen. Suppose player $\mathcal{A}$ chooses the $k$ th edge so that $G_{n, k} \in \mathcal{K}_{4}(z)$ with $z \equiv 0(\bmod 4)$. Then $P\left(G_{n, k}\right)=$ $\left\{u_{1}\right\}$ and $T\left(G_{n, k}\right)=\left\{u_{2}\right\}$, with $\left(u_{1}, u_{2}\right) \in E_{k}$. The strategy described here will work for $\mathcal{B}$ as well. We proceed by induction.

Base case: $z=0$. All vertices are out of play except $u_{1}$ and $u_{2}$, which are already connected by an edge. Since no new edge can be chosen, $\mathcal{A}$ wins.

Assume for induction that the statement holds when $z=4 m$ for some $m \geq 0$. Suppose $z=4(m+1)$. Let $Z\left(G_{n, k}\right)=\left\{w_{1}, \ldots, w_{4 m+4}\right\}$. Each vertex $v \notin\left(Z\left(G_{n, k}\right) \cup P\left(G_{n, k}\right) \cup\right.$ $\left.T\left(G_{n, k}\right)\right)$ has $\operatorname{deg}_{G_{n, k}}(v)=3$ and is out of play.

Up to isomorphism, $\mathcal{B}$ has two choices: $\left(u_{1}, w_{1}\right)$ or $\left(u_{2}, w_{1}\right)$. $\mathcal{A}$ chooses whichever edge $\mathcal{B}$ did not. $\mathcal{B}$ must choose $\left(u_{1}, w_{2}\right)$. $\mathcal{A}$ chooses $\left(w_{1}, w_{2}\right)$. $\mathcal{B}$ must choose $\left(w_{2}, w_{3}\right)$. $\mathcal{A}$ chooses $\left(w_{3}, w_{4}\right)$. Now vertices $u_{1}, u_{2}, w_{1}, w_{2}$ are out of play, $z\left(G_{n, k+6}\right)=4 m, p\left(G_{n, k+6}\right)=1$, and $t\left(G_{n, k+6}\right)=1$ with $P\left(G_{n, k+6}\right)=\left\{w_{4}\right\}, T\left(G_{n, k+6}\right)=\left\{w_{3}\right\},\left(w_{3}, w_{4}\right) \in E_{k+6}$. Thus $G_{n, k+6} \in \mathcal{K}_{4}(4 m)$, so $\mathcal{A}$ wins by induction.

Theorem 4.5. For $n \geq 5, f_{\{B 3, C\}}(n)=\mathcal{B} \Longleftrightarrow n \equiv 2(\bmod 4)$.

Proof. For small values of $n$, an exhaustive case analysis can be carried out by hand calculation.

\begin{tabular}{c|c|c|c|c|c|c}
$n$ & 3 & 4 & 5 & 6 & 7 & 8 \\
\hline$f_{\{B 3, C\}}(n)$ & $\mathcal{A}$ & $\mathcal{B}$ & $\mathcal{A}$ & $\mathcal{B}$ & $\mathcal{A}$ & $\mathcal{A}$
\end{tabular}




\section{$n \equiv 0(\bmod 4):$}

We proceed by induction on $n$.

Base case: $n=8$. This was solved by hand calculation.

Assume for induction that $f_{\{B 3, C\}}(n)=\mathcal{A}$ when $n=4 m$ for some $m \geq 2$. Suppose $n=4 m+4 . V=\left\{v_{1}, \ldots, v_{4 m+4}\right\}$.

$\mathcal{A}$ chooses $\left(v_{1}, v_{2}\right)$. $\mathcal{B}$ must choose $\left(v_{1}, v_{3}\right)$. $\mathcal{A}$ chooses $\left(v_{2}, v_{3}\right)$ to make a triangle. $\mathcal{B}$ must choose $\left(v_{1}, v_{4}\right)$. $\mathcal{A}$ chooses $\left(v_{2}, v_{4}\right)$. $\mathcal{B}$ has two choices: $\left(v_{3}, v_{4}\right)$ or $\left(v_{3}, v_{5}\right)$.

- If $\mathcal{B}$ chooses $\left(v_{3}, v_{4}\right)$, then vertices $v_{1}, v_{2}, v_{3}, v_{4}$ are out of play. The remaining vertices $v_{5}, \ldots, v_{4 m+4}$ have degree zero and it is $\mathcal{A}$ 's turn. Play continues as in $\Gamma_{B 3, C}(4 m)$, so $\mathcal{A}$ wins by induction.

- If $\mathcal{B}$ chooses $\left(v_{3}, v_{5}\right)$, then $\mathcal{A}$ chooses $\left(v_{4}, v_{5}\right)$. Now vertices $v_{1}, v_{2}, v_{3}, v_{4}$ are out of play. $\mathcal{B}$ must choose $\left(v_{5}, v_{6}\right)$. $\mathcal{A}$ chooses $\left(v_{6}, v_{7}\right)$. $\mathcal{B}$ has two choices: $\left(v_{6}, v_{8}\right)$ or $\left(v_{7}, v_{8}\right)$. $\mathcal{A}$ chooses whichever edge $\mathcal{B}$ did not. Now $v_{5}, v_{6}$ are also out of play. $z\left(G_{4 m+4,11}\right)=4 m-4, p\left(G_{4 m+4,11}\right)=0$, and $t\left(G_{4 m+4,11}\right)=2$ with $T\left(G_{4 m+4,11}\right)=$ $\left\{v_{7}, v_{8}\right\},\left(v_{7}, v_{8}\right) \in E_{11}$. Thus $G_{4 m+4,11} \in \mathcal{K}_{1}(4 m-4)$, so $\mathcal{A}$ wins by Lemma 4.1 .

\section{$n \equiv 1(\bmod 4):$}

We proceed by induction on $n$.

Base case: $n=5$. This was solved by hand calculation.

Assume for induction that $f_{\{B 3, C\}}(n)=\mathcal{A}$ when $n=4 m+1$ for some $m \geq 1$. Suppose $n=4 m+5 . V=\left\{v_{1}, \ldots, v_{4 m+5}\right\}$.

$\mathcal{A}$ chooses $\left(v_{1}, v_{2}\right)$. $\mathcal{B}$ must choose $\left(v_{1}, v_{3}\right)$. $\mathcal{A}$ chooses $\left(v_{2}, v_{3}\right)$ to make a triangle. $\mathcal{B}$ must choose $\left(v_{1}, v_{4}\right)$. $\mathcal{A}$ chooses $\left(v_{2}, v_{4}\right)$. $\mathcal{B}$ has two choices: $\left(v_{3}, v_{4}\right)$ or $\left(v_{3}, v_{5}\right)$.

- If $\mathcal{B}$ chooses $\left(v_{3}, v_{4}\right)$, then vertices $v_{1}, v_{2}, v_{3}, v_{4}$ are out of play. The remaining vertices $v_{5}, \ldots, v_{4 m+5}$ have degree zero and it is $\mathcal{A}$ 's turn. Play continues as in $\Gamma_{B 3, C}(4 m+1)$, so $\mathcal{A}$ wins by induction.

- If $\mathcal{B}$ chooses $\left(v_{3}, v_{5}\right)$, then $\mathcal{A}$ chooses $\left(v_{4}, v_{5}\right)$. Now vertices $v_{1}, v_{2}, v_{3}, v_{4}$ are out of play. $z\left(G_{4 m+5,7}\right)=4 m, p\left(G_{4 m+5,7}\right)=0$, and $t\left(G_{4 m+5,7}\right)=1$. Thus $G_{4 m+5,7} \in \mathcal{K}_{2}(4 m)$, so $\mathcal{A}$ wins by Lemma 4.2 .

\section{$n \equiv 2(\bmod 4):$}

Suppose $n=4 m+2$ for some $m \geq 1$. $V=\left\{v_{1}, \ldots, v_{4 m+2}\right\}$. We give a winning strategy for player $\mathcal{B}$.

$\mathcal{A}$ must choose $\left(v_{1}, v_{2}\right)$. $\mathcal{B}$ chooses $\left(v_{1}, v_{3}\right)$. $\mathcal{A}$ has three choices: $\left(v_{1}, v_{4}\right),\left(v_{2}, v_{3}\right)$, or $\left(v_{2}, v_{4}\right)$.

- If $\mathcal{A}$ chooses $\left(v_{1}, v_{4}\right)$ then $\mathcal{B}$ chooses $\left(v_{2}, v_{3}\right)$.

- If $\mathcal{A}$ chooses one of $\left(v_{2}, v_{3}\right)$ or $\left(v_{2}, v_{4}\right)$, then $\mathcal{B}$ chooses $\left(v_{1}, v_{4}\right)$.

The three possible resulting graphs are isomorphic, so without loss of generality, consider the first case. $\mathcal{A}$ again has three choices: $\left(v_{2}, v_{4}\right),\left(v_{2}, v_{5}\right)$, or $\left(v_{4}, v_{5}\right)$. 
- If $\mathcal{A}$ chooses $\left(v_{2}, v_{4}\right)$ then $\mathcal{B}$ chooses $\left(v_{3}, v_{5}\right)$.

- If $\mathcal{A}$ chooses $\left(v_{2}, v_{5}\right)$ then $\mathcal{B}$ chooses $\left(v_{3}, v_{4}\right)$.

- If $\mathcal{A}$ chooses $\left(v_{4}, v_{5}\right)$ then $\mathcal{B}$ chooses $\left(v_{2}, v_{4}\right)$.

As above, the three resulting graphs are isomorphic. Without loss of generality, consider the first case. $\mathcal{A}$ has three choices: $\left(v_{4}, v_{5}\right),\left(v_{4}, v_{6}\right)$, or $\left(v_{5}, v_{6}\right)$.

- If $\mathcal{A}$ chooses one of $\left(v_{4}, v_{5}\right)$ or $\left(v_{5}, v_{6}\right)$, then $\mathcal{B}$ chooses the other one. Now vertices $v_{1}, v_{2}, v_{3}, v_{4}, v_{5}$ are out of play. $z\left(G_{4 m+2,8}\right)=4 m-4, p\left(G_{4 m+2,8}\right)=1$, and $t\left(G_{4 m+2,8}\right)=0$. Thus $G_{4 m+2,8} \in \mathcal{K}_{3}(4 m-4)$, so $\mathcal{B}$ wins by Lemma 4.3 .

- If $\mathcal{A}$ chooses $\left(v_{4}, v_{6}\right)$ then $\mathcal{B}$ chooses $\left(v_{5}, v_{6}\right)$. Now vertices $v_{1}, v_{2}, v_{3}, v_{4}$ are out of play. $z\left(G_{4 m+2,8}\right)=4 m-4, p\left(G_{4 m+2,8}\right)=0$, and $t\left(G_{4 m+2,8}\right)=2$ with $T\left(G_{4 m+2,8}\right)=$ $\left\{v_{5}, v_{6}\right\},\left(v_{5}, v_{6}\right) \in E_{8}$. Thus $G_{4 m+2,8} \in \mathcal{K}_{1}(4 m-4)$, so $\mathcal{B}$ wins by Lemma 4.1.

\section{$n \equiv 3(\bmod 4):$}

We proceed by induction on $n$.

Base case: $n=7$. This was solved by hand calculation.

Assume for induction that $f_{\{B 3, C\}}(n)=\mathcal{A}$ when $n=4 m+3$ for some $m \geq 1$. Suppose $n=4 m+7 . V=\left\{v_{1}, \ldots, v_{4 m+7}\right\}$.

$\mathcal{A}$ chooses $\left(v_{1}, v_{2}\right)$. $\mathcal{B}$ must choose $\left(v_{1}, v_{3}\right)$. $\mathcal{A}$ chooses $\left(v_{2}, v_{3}\right)$ to make a triangle. $\mathcal{B}$ must choose $\left(v_{1}, v_{4}\right)$. $\mathcal{A}$ chooses $\left(v_{2}, v_{4}\right)$. $\mathcal{B}$ has two choices: $\left(v_{3}, v_{4}\right)$ or $\left(v_{3}, v_{5}\right)$.

- If $\mathcal{B}$ chooses $\left(v_{3}, v_{4}\right)$, then vertices $v_{1}, v_{2}, v_{3}, v_{4}$ are out of play. The remaining vertices $v_{5}, \ldots, v_{4 m+7}$ have degree zero and it is $\mathcal{A}$ 's turn. Play continues as in $\Gamma_{B 3, C}(4 m+3)$, so $\mathcal{A}$ wins by induction.

- If $\mathcal{B}$ chooses $\left(v_{3}, v_{5}\right)$, then $\mathcal{A}$ chooses $\left(v_{4}, v_{5}\right)$. $\mathcal{B}$ must choose $\left(v_{5}, v_{6}\right)$. $\mathcal{A}$ chooses $\left(v_{6}, v_{7}\right)$. Vertices $v_{1}, v_{2}, v_{3}, v_{4}, v_{5}$ are out of play. $z\left(G_{4 m+7,9}\right)=4 m, p\left(G_{4 m+7,9}\right)=1$, and $t\left(G_{4 m+7,9}\right)=1$ with $P\left(G_{4 m+7,9}\right)=\left\{v_{7}\right\}$ and $T\left(G_{4 m+7,9}\right)=\left\{v_{6}\right\},\left(v_{6}, v_{7}\right) \in E_{9}$. Thus $G_{4 m+7,9} \in \mathcal{K}_{4}(4 m)$, so $\mathcal{A}$ wins by Lemma 4.4.

\section{$5 \mathcal{R}=\{B 3, T, C\}$}

In the game $\Gamma_{\{B 3, T, C\}}$, we define graph classes $\mathcal{L}_{i}(z)$ for $i=1,2,3$, where $z \geq 0$ is an integer. For large enough $n$, one player can force that $G_{n, k} \in \mathcal{L}_{1}(z)$ early in the game. A few rounds later, the same player can force that $G_{n, k} \in\left(\mathcal{L}_{2}(z) \cup \mathcal{L}_{3}(z)\right)$. From here, this player then maintains a periodic method of gameplay, continuously returning to graphs in $\mathcal{L}_{2}(z) \cup \mathcal{L}_{3}(z)$ after a fixed number of rounds. Following this strategy until the end of the game forces a win. 
For any graph $G(V, E)$, define the set $F(G)$ to be the set of edges in $E^{c}$ that will create a triangle if chosen. That is,

$$
F(G)=\left\{\left(v_{i}, v_{j}\right) \notin E \mid \exists w \in V \text { such that }\left(v_{i}, w\right),\left(v_{j}, w\right) \in E\right\} .
$$

Define sets of graphs $\mathcal{L}_{i}(z)$ for $i=1,2,3$ as follows:

1. For any integer $z \geq 0, G(V, E) \in \mathcal{L}_{1}(z) \Longleftrightarrow z(G)=z$ and ONE of the following holds:

(a) $p(G)=1, t(G)=1$, and if $u_{1} \in P(G), u_{2} \in T(G)$, then $\left(u_{1}, u_{2}\right) \in F(G)$,

(b) $p(G)=1, t(G)=1$, and if $u_{1} \in P(G), u_{2} \in T(G)$, then $\left(u_{1}, u_{2}\right) \notin(E \cup F(G))$,

(c) $p(G)=0, t(G)=3$, and, for some ordering of $T(G)$, if $u_{1}, u_{2}, u_{3} \in T(G)$, then $\left(u_{1}, u_{2}\right) \in E$ and $\left(u_{1}, u_{3}\right),\left(u_{2}, u_{3}\right) \in F(G)$, or

(d) $p(G)=0, t(G)=3$, and, for some ordering of $T(G)$, if $u_{1}, u_{2}, u_{3} \in T(G)$, then $\left(u_{1}, u_{2}\right) \in E,\left(u_{1}, u_{3}\right) \in F(G)$, and $\left(u_{2}, u_{3}\right) \notin(E \cup F(G))$.

2. For any integer $z \geq 0, G(V, E) \in \mathcal{L}_{2}(z) \Longleftrightarrow z(G)=z$ and ONE of the following holds:

(a) $p(G)=1, t(G)=1$, and if $u_{1} \in P(G), u_{2} \in T(G)$, then $\left(u_{1}, u_{2}\right) \in F(G)$,

(b) $p(G)=0, t(G)=2$, and if $u_{1}, u_{2} \in T(G)$, then $\left(u_{1}, u_{2}\right) \in E$, or

(c) $p(G)=0, t(G)=2$, and if $u_{1}, u_{2} \in T(G)$, then $\left(u_{1}, u_{2}\right) \in F(G)$.

3. For any integer $z \geq 0, G(V, E) \in \mathcal{L}_{3}(z) \Longleftrightarrow z(G)=z$ and ONE of the following holds:

(a) $p(G)=1$ and $t(G)=0$, or

(b) $p(G)=0, t(G)=3$, and, for some ordering of $T(G)$, if $u_{1}, u_{2}, u_{3} \in T(G)$, then $\left(u_{1}, u_{2}\right),\left(u_{1}, u_{3}\right) \in E$.

In the proof of the following Lemma, the set $\mathcal{L}_{i}(z)(j)$ refers to those graphs in $\mathcal{L}_{i}(z)$ that result from case $(j)$. For example, the set $\mathcal{L}_{1}(z)(a)$ consists of graphs in $\mathcal{L}_{1}(z)$ such that case $(a)$ is satisfied: For any integer $z \geq 0, G(V, E) \in \mathcal{L}_{1}(z)(a) \Longleftrightarrow z(G)=z$, $p(G)=1, t(G)=1$, and if $u_{1} \in P(G), u_{2} \in T(G)$, then $\left(u_{1}, u_{2}\right) \in F(G)$.

Lemma 5.1. In $\Gamma_{\{B 3, T, C\}}(n), \mathcal{A}$ can choose edges $e_{1}, e_{3}, \ldots, e_{k}$ so that:

- when $n \geq 11$ and $k=15, G_{n, 15} \in \mathcal{L}_{1}(n-11)$, or

- when $n \geq 15$ and $k=21, G_{n, 21} \in \mathcal{L}_{1}(n-15)$. 
Proof. Let $n \geq 11$. $\mathcal{A}$ chooses $\left(v_{1}, v_{2}\right)$. Up to isomorphism, $\mathcal{B}$ must choose $\left(v_{1}, v_{3}\right)$. $\mathcal{A}$ chooses $\left(v_{1}, v_{4}\right)$. $\mathcal{B}$ must choose $\left(v_{2}, v_{5}\right)$. $\mathcal{A}$ chooses $\left(v_{2}, v_{6}\right)$. $\mathcal{B}$ has two choices: $\left(v_{3}, v_{5}\right)$ or $\left(v_{3}, v_{7}\right)$. $\mathcal{A}$ chooses whichever edge $\mathcal{B}$ did not. Now $\mathcal{B}$ has six choices: $\left(v_{4}, v_{5}\right),\left(v_{4}, v_{6}\right)$, $\left(v_{4}, v_{8}\right),\left(v_{5}, v_{8}\right),\left(v_{6}, v_{7}\right)$, or $\left(v_{6}, v_{8}\right)$.

- If $\mathcal{B}$ chooses one of $\left(v_{4}, v_{5}\right)$ or $\left(v_{4}, v_{8}\right)$, then $\mathcal{A}$ chooses the other edge. $\mathcal{B}$ has two choices: $\left(v_{6}, v_{7}\right)$ or $\left(v_{6}, v_{9}\right)$. $\mathcal{A}$ chooses whichever edge $\mathcal{B}$ did not. $\mathcal{B}$ has five choices: $\left(v_{7}, v_{8}\right),\left(v_{7}, v_{10}\right),\left(v_{8}, v_{9}\right),\left(v_{8}, v_{10}\right)$, and $\left(v_{9}, v_{10}\right)$.

- If $\mathcal{B}$ chooses one of $\left(v_{7}, v_{8}\right)$ or $\left(v_{8}, v_{10}\right)$, then $\mathcal{A}$ chooses the other edge. $\mathcal{B}$ has two choices: $\left(v_{9}, v_{10}\right)$ or $\left(v_{9}, v_{11}\right)$. $\mathcal{A}$ chooses whichever edge $\mathcal{B}$ did not. The resulting graph is in $\mathcal{L}_{1}(n-11)(a)$.

- If $\mathcal{B}$ chooses one of $\left(v_{7}, v_{10}\right)$ or $\left(v_{8}, v_{9}\right)$, then $\mathcal{A}$ chooses the other edge. $\mathcal{B}$ has three choices: $\left(v_{8}, v_{10}\right),\left(v_{8}, v_{11}\right)$, or $\left(v_{10}, v_{11}\right)$. If $\mathcal{B}$ chooses $\left(v_{8}, v_{10}\right), \mathcal{A}$ chooses $\left(v_{9}, v_{11}\right)$. If $\mathcal{B}$ chooses $\left(v_{8}, v_{11}\right), \mathcal{A}$ chooses $\left(v_{9}, v_{10}\right)$. If $\mathcal{B}$ chooses $\left(v_{10}, v_{11}\right), \mathcal{A}$ chooses $\left(v_{8}, v_{10}\right)$. In each case, the resulting graph is in $\mathcal{L}_{1}(n-11)(b)$.

- If $\mathcal{B}$ chooses $\left(v_{9}, v_{10}\right)$, then $\mathcal{A}$ chooses $\left(v_{7}, v_{10}\right) . \mathcal{B}$ has three choices: $\left(v_{8}, v_{9}\right)$, $\left(v_{8}, v_{11}\right)$, or $\left(v_{9}, v_{11}\right)$. If $\mathcal{B}$ chooses one of $\left(v_{8}, v_{9}\right)$ or $\left(v_{8}, v_{11}\right), \mathcal{A}$ chooses the other edge. If $\mathcal{B}$ chooses $\left(v_{9}, v_{11}\right), \mathcal{A}$ chooses $\left(v_{8}, v_{10}\right)$. In each case, the resulting graph is in $\mathcal{L}_{1}(n-11)(b)$.

- If $\mathcal{B}$ chooses one of $\left(v_{4}, v_{6}\right)$ or $\left(v_{5}, v_{8}\right)$, then $\mathcal{A}$ chooses the other edge.

If $\mathcal{B}$ chooses one of $\left(v_{6}, v_{7}\right)$ or $\left(v_{6}, v_{8}\right)$, then $\mathcal{A}$ chooses $\left(v_{5}, v_{8}\right)$.

The resulting three graphs have:

1. $n-8$ vertices of degree 0 , two vertices of degree 1 , and two vertices of degree 2 ,

2. the two vertices of degree 2 are connected by an edge, and

3. for each pair of vertices $v_{i}$ and $v_{j}$ of degree 1 or 2 , not both of degree 2 , the distance between $v_{i}$ and $v_{j}$ is at least 3 .

Gameplay is identical in each case, so we may assume the first case. $\mathcal{B}$ has four choices: $\left(v_{4}, v_{7}\right),\left(v_{4}, v_{9}\right),\left(v_{7}, v_{8}\right)$, or $\left(v_{7}, v_{9}\right)$.

- If $\mathcal{B}$ chooses $\left(v_{4}, v_{7}\right)$, then $\mathcal{A}$ chooses $\left(v_{6}, v_{9}\right)$.

If $\mathcal{B}$ chooses $\left(v_{4}, v_{9}\right)$, then $\mathcal{A}$ chooses $\left(v_{6}, v_{7}\right)$.

If $\mathcal{B}$ chooses $\left(v_{7}, v_{9}\right)$, then $\mathcal{A}$ chooses $\left(v_{4}, v_{7}\right)$.

The resulting three graphs have:

1. $n-9$ vertices of degree 0 , two vertices of degree 1 , and one vertex of degree 2 , and 
2. for each pair of vertices $v_{i}$ and $v_{j}$ of degree 1 or 2 , the distance between $v_{i}$ and $v_{j}$ is at least 3 .

Gameplay is identical in each case, so we may consider the first case. $\mathcal{B}$ has four choices: $\left(v_{7}, v_{8}\right),\left(v_{7}, v_{10}\right),\left(v_{8}, v_{9}\right)$, or $\left(v_{8}, v_{10}\right)$.

* If $\mathcal{B}$ chooses one of $\left(v_{7}, v_{8}\right)$ or $\left(v_{8}, v_{10}\right)$, then $\mathcal{A}$ chooses the other edge. $\mathcal{B}$ has two choices: $\left(v_{9}, v_{10}\right)$ or $\left(v_{9}, v_{11}\right)$. $\mathcal{A}$ chooses whichever edge $\mathcal{B}$ did not. The resulting graph is in $\mathcal{L}_{1}(n-11)(a)$.

* If $\mathcal{B}$ chooses one of $\left(v_{7}, v_{10}\right)$ or $\left(v_{8}, v_{9}\right)$, then $\mathcal{A}$ chooses the other edge. $\mathcal{B}$ has three choices: $\left(v_{8}, v_{10}\right),\left(v_{8}, v_{11}\right)$, or $\left(v_{10}, v_{11}\right)$. If $\mathcal{B}$ chooses $\left(v_{8}, v_{10}\right), \mathcal{A}$ chooses $\left(v_{9}, v_{11}\right)$. If $\mathcal{B}$ chooses $\left(v_{8}, v_{11}\right), \mathcal{A}$ chooses $\left(v_{9}, v_{10}\right)$. If $\mathcal{B}$ chooses $\left(v_{10}, v_{11}\right), \mathcal{A}$ chooses $\left(v_{8}, v_{10}\right)$. In each case, the resulting graph is in $\mathcal{L}_{1}(n-$ 11) (b).

- If $\mathcal{B}$ chooses $\left(v_{7}, v_{8}\right)$, then $\mathcal{A}$ chooses $\left(v_{4}, v_{9}\right) . \mathcal{B}$ has five choices: $\left(v_{6}, v_{7}\right)$, $\left(v_{6}, v_{10}\right),\left(v_{7}, v_{9}\right),\left(v_{7}, v_{10}\right)$, or $\left(v_{9}, v_{10}\right)$.

* If $\mathcal{B}$ chooses $\left(v_{6}, v_{7}\right)$, then $\mathcal{A}$ chooses $\left(v_{8}, v_{10}\right)$. $\mathcal{B}$ has two choices: $\left(v_{9}, v_{10}\right)$ or $\left(v_{9}, v_{11}\right)$. $\mathcal{A}$ chooses whichever edge $\mathcal{B}$ did not. The resulting graph is in $\mathcal{L}_{1}(n-11)(a)$.

* If $\mathcal{B}$ chooses one of $\left(v_{6}, v_{10}\right)$ or $\left(v_{7}, v_{9}\right)$, then $\mathcal{A}$ chooses the other edge. $\mathcal{B}$ has three choices: $\left(v_{8}, v_{10}\right),\left(v_{8}, v_{11}\right)$, or $\left(v_{10}, v_{11}\right)$. If $\mathcal{B}$ chooses $\left(v_{8}, v_{10}\right), \mathcal{A}$ chooses $\left(v_{9}, v_{11}\right)$. If $\mathcal{B}$ chooses $\left(v_{8}, v_{11}\right), \mathcal{A}$ chooses $\left(v_{9}, v_{10}\right)$. If $\mathcal{B}$ chooses $\left(v_{10}, v_{11}\right), \mathcal{A}$ chooses $\left(v_{8}, v_{10}\right)$. In each case, the resulting graph is in $\mathcal{L}_{1}(n-$ $11)(b)$.

* If $\mathcal{B}$ chooses one of $\left(v_{7}, v_{10}\right)$ or $\left(v_{9}, v_{10}\right)$, then $\mathcal{A}$ chooses the other edge. $\mathcal{B}$ has four choices: $\left(v_{6}, v_{8}\right),\left(v_{6}, v_{10}\right),\left(v_{6}, v_{11}\right)$, or $\left(v_{9}, v_{11}\right)$.

- If $\mathcal{B}$ chooses one of $\left(v_{6}, v_{8}\right)$ or $\left(v_{9}, v_{11}\right)$, then $\mathcal{A}$ chooses the other edge. The resulting graph is in $\mathcal{L}_{1}(n-11)(a)$.

- If $\mathcal{B}$ chooses one of $\left(v_{6}, v_{10}\right)$ or $\left(v_{6}, v_{11}\right)$, then $\mathcal{A}$ chooses the other edge. The resulting graph is in $\mathcal{L}_{1}(n-11)(b)$.

Now let $n \geq 15$. $\mathcal{A}$ chooses $\left(v_{1}, v_{2}\right)$. Up to isomorphism, $\mathcal{B}$ must choose $\left(v_{1}, v_{3}\right)$. $\mathcal{A}$ chooses $\left(v_{1}, v_{4}\right)$. $\mathcal{B}$ must choose $\left(v_{2}, v_{5}\right)$. $\mathcal{A}$ chooses $\left(v_{3}, v_{5}\right)$. $\mathcal{B}$ has four choices: $\left(v_{2}, v_{6}\right)$, $\left(v_{4}, v_{5}\right),\left(v_{4}, v_{6}\right)$, or $\left(v_{5}, v_{6}\right)$.

- If $\mathcal{B}$ chooses one of $\left(v_{2}, v_{6}\right)$ or $\left(v_{4}, v_{6}\right)$, then $\mathcal{A}$ chooses the other edge. Now $\mathcal{B}$ has two choices: $\left(v_{3}, v_{6}\right)$ or $\left(v_{3}, v_{7}\right)$.

- If $\mathcal{B}$ chooses $\left(v_{3}, v_{6}\right)$, then $\mathcal{A}$ chooses $\left(v_{4}, v_{7}\right)$. Call this graph $G_{(1)}^{1}$.

- If $\mathcal{B}$ chooses $\left(v_{3}, v_{7}\right)$, then $\mathcal{A}$ chooses $\left(v_{4}, v_{5}\right)$. This graph is isomorphic to $G_{(1)}^{1}$.

- If $\mathcal{B}$ chooses $\left(v_{4}, v_{5}\right)$, then $\mathcal{A}$ chooses $\left(v_{2}, v_{6}\right)$. Now $\mathcal{B}$ has three choices: $\left(v_{3}, v_{6}\right)$, $\left(v_{3}, v_{7}\right)$, or $\left(v_{6}, v_{7}\right)$. 
- If $\mathcal{B}$ chooses $\left(v_{3}, v_{6}\right)$, then $\mathcal{A}$ chooses $\left(v_{4}, v_{7}\right)$. This graph is isomorphic to $G_{(1)}^{1}$.

- If $\mathcal{B}$ chooses $\left(v_{3}, v_{7}\right)$, then $\mathcal{A}$ chooses $\left(v_{4}, v_{6}\right)$. This graph is isomorphic to $G_{(1)}^{1}$.

- If $\mathcal{B}$ chooses $\left(v_{6}, v_{7}\right)$, then $\mathcal{A}$ chooses $\left(v_{3}, v_{6}\right)$. This graph is isomorphic to $G_{(1)}^{1}$.

- If $\mathcal{B}$ chooses $\left(v_{5}, v_{6}\right)$, then $\mathcal{A}$ chooses $\left(v_{4}, v_{6}\right)$. Now $\mathcal{B}$ has two choices: $\left(v_{2}, v_{7}\right)$ or $\left(v_{4}, v_{7}\right)$. $\mathcal{A}$ chooses whichever edge $\mathcal{B}$ did not. Call this graph $G_{(2)}^{1}$.

We can now examine the two graphs $G_{(1)}^{1}$ and $G_{(2)}^{1}$.

Case 1.1: $G_{n, 9}=G_{(1)}^{1}$. $\mathcal{B}$ has three choices: $\left(v_{5}, v_{7}\right),\left(v_{5}, v_{8}\right)$, or $\left(v_{7}, v_{8}\right)$.

- If $\mathcal{B}$ chooses $\left(v_{5}, v_{7}\right)$, then $\mathcal{A}$ chooses $\left(v_{7}, v_{8}\right)$. Call this graph $G_{(1)}^{2}$.

- If $\mathcal{B}$ chooses one of $\left(v_{5}, v_{8}\right)$ or $\left(v_{7}, v_{8}\right)$, then $\mathcal{A}$ chooses the other edge. Call this graph $G_{(2)}^{2}$.

Case 1.2: $G_{n, 9}=G_{(2)}^{1}$. $\mathcal{B}$ has three choices: $\left(v_{3}, v_{7}\right),\left(v_{3}, v_{8}\right)$, or $\left(v_{6}, v_{8}\right)$.

- If $\mathcal{B}$ chooses one of $\left(v_{3}, v_{7}\right)$ or $\left(v_{6}, v_{8}\right)$, then $\mathcal{A}$ chooses the other edge. This graph is isomorphic to $G_{(1)}^{2}$.

- If $\mathcal{B}$ chooses $\left(v_{3}, v_{8}\right)$, then $\mathcal{A}$ chooses $\left(v_{6}, v_{9}\right)$. Call this graph $G_{(3)}^{2}$.

Now we look at the three graphs $G_{(i)}^{2}$, for $i=1,2,3$.

Case 2.1: $G_{n, 11}=G_{(1)}^{2}$. $\mathcal{B}$ must choose $\left(v_{8}, v_{9}\right)$. $\mathcal{A}$ chooses $\left(v_{8}, v_{10}\right)$. $\mathcal{B}$ must choose $\left(v_{9}, v_{11}\right)$. $\mathcal{A}$ chooses $\left(v_{10}, v_{11}\right)$. $\mathcal{B}$ has two choices: $\left(v_{9}, v_{12}\right)$ or $\left(v_{11}, v_{12}\right)$.

If $\mathcal{B}$ chooses $\left(v_{9}, v_{12}\right), \mathcal{A}$ chooses $\left(v_{11}, v_{13}\right)$.

If $\mathcal{B}$ chooses $\left(v_{11}, v_{12}\right), \mathcal{A}$ chooses $\left(v_{9}, v_{13}\right)$.

The resulting two graphs are isomorphic, so without loss of generality we may consider the first case. $\mathcal{B}$ has five choices: $\left(v_{10}, v_{12}\right),\left(v_{10}, v_{14}\right),\left(v_{12}, v_{13}\right),\left(v_{12}, v_{14}\right)$, or $\left(v_{13}, v_{14}\right)$.

- If $\mathcal{B}$ chooses one of $\left(v_{10}, v_{12}\right)$ or $\left(v_{12}, v_{14}\right)$, then $\mathcal{A}$ chooses the other edge. $\mathcal{B}$ has two choices: $\left(v_{13}, v_{14}\right)$ or $\left(v_{13}, v_{15}\right)$. $\mathcal{A}$ chooses whichever edge $\mathcal{B}$ did not. The resulting graph is in $\mathcal{L}_{1}(n-15)(a)$.

- If $\mathcal{B}$ chooses one of $\left(v_{10}, v_{14}\right)$ or $\left(v_{12}, v_{13}\right)$, then $\mathcal{A}$ chooses the other edge. $\mathcal{B}$ has three choices: $\left(v_{12}, v_{14}\right),\left(v_{12}, v_{15}\right)$, or $\left(v_{14}, v_{15}\right)$.

If $\mathcal{B}$ chooses $\left(v_{12}, v_{14}\right)$, then $\mathcal{A}$ chooses $\left(v_{13}, v_{15}\right)$.

If $\mathcal{B}$ chooses $\left(v_{12}, v_{15}\right)$, then $\mathcal{A}$ chooses $\left(v_{13}, v_{14}\right)$.

If $\mathcal{B}$ chooses $\left(v_{14}, v_{15}\right)$, then $\mathcal{A}$ chooses $\left(v_{12}, v_{14}\right)$.

The resulting three graphs are in $\mathcal{L}_{1}(n-15)(b)$. 
- If $\mathcal{B}$ chooses $\left(v_{13}, v_{14}\right)$, then $\mathcal{A}$ chooses $\left(v_{10}, v_{14}\right)$. $\mathcal{B}$ has three choices: $\left(v_{12}, v_{13}\right)$, $\left(v_{12}, v_{15}\right)$, or $\left(v_{13}, v_{15}\right)$.

If $\mathcal{B}$ chooses one of $\left(v_{12}, v_{13}\right)$ or $\left(v_{12}, v_{15}\right)$, then $\mathcal{A}$ chooses the other edge.

If $\mathcal{B}$ chooses $\left(v_{13}, v_{15}\right)$, then $\mathcal{A}$ chooses $\left(v_{12}, v_{14}\right)$.

The resulting two graphs are in $\mathcal{L}_{1}(n-15)(b)$.

Case 2.2: $G_{n, 11}=G_{(2)}^{2} . \mathcal{B}$ must choose $\left(v_{7}, v_{9}\right) . \mathcal{A}$ chooses $\left(v_{8}, v_{10}\right) . \quad \mathcal{B}$ has two choices: $\left(v_{9}, v_{10}\right)$ or $\left(v_{9}, v_{11}\right)$. $\mathcal{A}$ chooses whichever edge $\mathcal{B}$ did not. $\mathcal{B}$ has two choices: $\left(v_{10}, v_{12}\right)$ or $\left(v_{11}, v_{12}\right)$. $\mathcal{A}$ chooses whichever edge $\mathcal{B}$ did not. $\mathcal{B}$ must choose $\left(v_{11}, v_{13}\right)$. $\mathcal{A}$ chooses $\left(v_{12}, v_{14}\right)$. $\mathcal{B}$ has two choices: $\left(v_{13}, v_{14}\right)$ or $\left(v_{13}, v_{15}\right)$. $\mathcal{A}$ chooses whichever edge $\mathcal{B}$ did not. The resulting graph is in $\mathcal{L}_{1}(n-15)(a)$.

Case 2.3: $G_{n, 11}=G_{(3)}^{2}$. $\mathcal{B}$ has four choices: $\left(v_{7}, v_{8}\right),\left(v_{7}, v_{10}\right),\left(v_{8}, v_{9}\right)$, or $\left(v_{8}, v_{10}\right)$.

Subcase 2.3.1: If $\mathcal{B}$ chooses one of $\left(v_{7}, v_{8}\right)$ or $\left(v_{8}, v_{9}\right)$, then $\mathcal{A}$ chooses the other edge. $\mathcal{B}$ must choose $\left(v_{9}, v_{10}\right)$. $\mathcal{A}$ chooses $\left(v_{10}, v_{11}\right)$. $\mathcal{B}$ has two choices: $\left(v_{10}, v_{12}\right)$ or $\left(v_{11}, v_{12}\right)$.

If $\mathcal{B}$ chooses $\left(v_{10}, v_{12}\right)$, then $\mathcal{A}$ chooses $\left(v_{11}, v_{13}\right)$.

If $\mathcal{B}$ chooses $\left(v_{11}, v_{12}\right)$, then $\mathcal{A}$ chooses $\left(v_{10}, v_{13}\right)$.

The resulting two graphs are isomorphic, so without loss of generality we may consider the first case. $\mathcal{B}$ has four choices: $\left(v_{11}, v_{14}\right),\left(v_{12}, v_{13}\right),\left(v_{12}, v_{14}\right)$, or $\left(v_{13}, v_{14}\right)$.

- If $\mathcal{B}$ chooses one of $\left(v_{11}, v_{14}\right)$ or $\left(v_{12}, v_{13}\right)$, then $\mathcal{A}$ chooses the other edge. $\mathcal{B}$ has four choices: $\left(v_{12}, v_{14}\right),\left(v_{12}, v_{15}\right),\left(v_{13}, v_{15}\right)$, or $\left(v_{14}, v_{15}\right)$.

- If $\mathcal{B}$ chooses one of $\left(v_{12}, v_{14}\right)$ or $\left(v_{13}, v_{15}\right)$, then $\mathcal{A}$ chooses the other edge. The resulting graph is in $\mathcal{L}_{1}(n-15)(b)$.

- If $\mathcal{B}$ chooses one of $\left(v_{12}, v_{15}\right)$ or $\left(v_{14}, v_{15}\right)$, then $\mathcal{A}$ chooses the other edge. The resulting graph is in $\mathcal{L}_{1}(n-15)(c)$.

- If $\mathcal{B}$ chooses $\left(v_{12}, v_{14}\right)$, then $\mathcal{A}$ chooses $\left(v_{11}, v_{14}\right)$. $\mathcal{B}$ has four choices: $\left(v_{12}, v_{13}\right)$, $\left(v_{12}, v_{15}\right),\left(v_{13}, v_{15}\right)$, or $\left(v_{14}, v_{15}\right)$.

- If $\mathcal{B}$ chooses one of $\left(v_{12}, v_{13}\right)$ or $\left(v_{13}, v_{15}\right)$, then $\mathcal{A}$ chooses the other edge. The resulting graph is in $\mathcal{L}_{1}(n-15)(b)$.

- If $\mathcal{B}$ chooses $\left(v_{12}, v_{15}\right)$, then $\mathcal{A}$ chooses $\left(v_{13}, v_{15}\right)$. The resulting graph is in $\mathcal{L}_{1}(n-15)(c)$.

- If $\mathcal{B}$ chooses $\left(v_{14}, v_{15}\right)$, then $\mathcal{A}$ chooses $\left(v_{13}, v_{15}\right)$. The resulting graph is in $\mathcal{L}_{1}(n-15)(d)$.

- If $\mathcal{B}$ chooses $\left(v_{13}, v_{14}\right)$, then $\mathcal{A}$ chooses $\left(v_{12}, v_{13}\right)$. $\mathcal{B}$ has two choices: $\left(v_{11}, v_{15}\right)$ or $\left(v_{14}, v_{15}\right)$. $\mathcal{A}$ chooses whichever edge $\mathcal{B}$ did not. The resulting graph is in $\mathcal{L}_{1}(n-$ $15)(d)$. 
Subcase 2.3.2: If $\mathcal{B}$ chooses $\left(v_{7}, v_{10}\right)$, then $\mathcal{A}$ chooses $\left(v_{8}, v_{11}\right)$.

If $\mathcal{B}$ chooses $\left(v_{8}, v_{10}\right)$, then $\mathcal{A}$ chooses $\left(v_{7}, v_{11}\right)$.

The two resulting graphs are isomorphic, so without loss of generality we may consider the first case. $\mathcal{B}$ has six choices: $\left(v_{8}, v_{9}\right),\left(v_{8}, v_{12}\right),\left(v_{9}, v_{10}\right),\left(v_{9}, v_{11}\right),\left(v_{9}, v_{12}\right)$, or $\left(v_{11}, v_{12}\right)$.

- If $\mathcal{B}$ chooses one of $\left(v_{8}, v_{9}\right)$ or $\left(v_{9}, v_{12}\right)$, then $\mathcal{A}$ chooses the other edge. Call this graph $G_{(1)}^{3}$.

- If $\mathcal{B}$ chooses one of $\left(v_{8}, v_{12}\right)$ or $\left(v_{9}, v_{10}\right)$, then $\mathcal{A}$ chooses the other edge. Call this graph $G_{(2)}^{3}$.

- If $\mathcal{B}$ chooses one of $\left(v_{9}, v_{11}\right)$ or $\left(v_{11}, v_{12}\right)$, then $\mathcal{A}$ chooses the other edge. Call this graph $G_{(3)}^{3}$.

All that remains is for us to look at the three graphs $G_{(i)}^{3}$, for $i=1,2,3$.

Case 3.1: $G_{n, 15}=G_{(1)}^{3}$. $\mathcal{B}$ has two choices: $\left(v_{10}, v_{11}\right)$ or $\left(v_{10}, v_{13}\right)$. $\mathcal{A}$ chooses whichever edge $\mathcal{B}$ did not. $\mathcal{B}$ has five choices: $\left(v_{11}, v_{13}\right),\left(v_{11}, v_{14}\right),\left(v_{12}, v_{13}\right),\left(v_{12}, v_{14}\right)$, or $\left(v_{13}, v_{14}\right)$.

- If $\mathcal{B}$ chooses one of $\left(v_{11}, v_{13}\right)$ or $\left(v_{13}, v_{14}\right)$, then $\mathcal{A}$ chooses the other edge. $\mathcal{B}$ has two choices: $\left(v_{12}, v_{14}\right)$ or $\left(v_{12}, v_{15}\right)$. $\mathcal{A}$ chooses whichever edge $\mathcal{B}$ did not. The resulting graph is in $\mathcal{L}_{1}(n-15)(a)$.

- If $\mathcal{B}$ chooses one of $\left(v_{11}, v_{14}\right)$ or $\left(v_{12}, v_{13}\right)$, then $\mathcal{A}$ chooses the other edge. $\mathcal{B}$ has three choices: $\left(v_{12}, v_{14}\right),\left(v_{12}, v_{15}\right)$, or $\left(v_{14}, v_{15}\right)$.

- If $\mathcal{B}$ chooses $\left(v_{12}, v_{14}\right)$, then $\mathcal{A}$ chooses $\left(v_{13}, v_{15}\right)$. The resulting graph is in $\mathcal{L}_{1}(n-15)(b)$.

- If $\mathcal{B}$ chooses one of $\left(v_{12}, v_{15}\right)$ or $\left(v_{14}, v_{15}\right)$, then $\mathcal{A}$ chooses the other edge. The resulting graph is in $\mathcal{L}_{1}(n-15)(d)$.

- If $\mathcal{B}$ chooses $\left(v_{12}, v_{14}\right)$, then $\mathcal{A}$ chooses $\left(v_{11}, v_{14}\right)$. $\mathcal{B}$ has three choices: $\left(v_{12}, v_{13}\right)$, $\left(v_{12}, v_{15}\right)$, or $\left(v_{13}, v_{15}\right)$.

- If $\mathcal{B}$ chooses $\left(v_{12}, v_{13}\right)$, then $\mathcal{A}$ chooses $\left(v_{13}, v_{15}\right)$. The resulting graph is in $\mathcal{L}_{1}(n-15)(b)$.

- If $\mathcal{B}$ chooses one of $\left(v_{12}, v_{15}\right)$ or $\left(v_{13}, v_{15}\right)$, then $\mathcal{A}$ chooses the other edge. The resulting graph is in $\mathcal{L}_{1}(n-15)(d)$.

Case 3.2: $G_{n, 15}=G_{(2)}^{3}$. $\mathcal{B}$ has three choices: $\left(v_{9}, v_{11}\right),\left(v_{9}, v_{13}\right)$, or $\left(v_{11}, v_{13}\right)$.

Subcase 3.2.1: If $\mathcal{B}$ chooses one of $\left(v_{9}, v_{11}\right)$ or $\left(v_{11}, v_{13}\right)$, then $\mathcal{A}$ chooses the other edge. $\mathcal{B}$ has five choices: $\left(v_{10}, v_{12}\right),\left(v_{10}, v_{14}\right),\left(v_{12}, v_{13}\right)$, or $\left(v_{12}, v_{14}\right)$.

- If $\mathcal{B}$ chooses one of $\left(v_{10}, v_{12}\right)$ or $\left(v_{12}, v_{14}\right)$, then $\mathcal{A}$ chooses the other edge. $\mathcal{B}$ has two choices: $\left(v_{13}, v_{14}\right)$ or $\left(v_{13}, v_{15}\right)$. $\mathcal{A}$ chooses whichever edge $\mathcal{B}$ did not. The resulting graph is in $\mathcal{L}_{1}(n-15)(a)$. 
- If $\mathcal{B}$ chooses one of $\left(v_{10}, v_{14}\right)$ or $\left(v_{12}, v_{13}\right)$, then $\mathcal{A}$ chooses the other edge. $\mathcal{B}$ has three choices: $\left(v_{12}, v_{14}\right),\left(v_{12}, v_{15}\right)$, or $\left(v_{14}, v_{15}\right)$.

If $\mathcal{B}$ chooses $\left(v_{12}, v_{14}\right)$, then $\mathcal{A}$ chooses $\left(v_{13}, v_{15}\right)$.

If $\mathcal{B}$ chooses $\left(v_{12}, v_{15}\right)$, then $\mathcal{A}$ chooses $\left(v_{13}, v_{14}\right)$.

If $\mathcal{B}$ chooses $\left(v_{14}, v_{15}\right)$, then $\mathcal{A}$ chooses $\left(v_{12}, v_{14}\right)$.

The resulting three graphs are in $\mathcal{L}_{1}(n-15)(b)$.

Subcase 3.2.2: If $\mathcal{B}$ chooses $\left(v_{9}, v_{13}\right)$, then $\mathcal{A}$ chooses $\left(v_{10}, v_{11}\right)$. $\mathcal{B}$ has five choices: $\left(v_{11}, v_{13}\right),\left(v_{11}, v_{14}\right),\left(v_{12}, v_{13}\right),\left(v_{12}, v_{14}\right)$, or $\left(v_{13}, v_{14}\right)$.

- If $\mathcal{B}$ chooses one of $\left(v_{11}, v_{13}\right)$ or $\left(v_{13}, v_{14}\right)$, then $\mathcal{A}$ chooses the other edge. $\mathcal{B}$ has two choices: $\left(v_{12}, v_{14}\right)$ or $\left(v_{12}, v_{15}\right)$. $\mathcal{A}$ chooses whichever edge $\mathcal{B}$ did not. The resulting graph is in $\mathcal{L}_{1}(n-15)(a)$.

- If $\mathcal{B}$ chooses one of $\left(v_{11}, v_{14}\right)$ or $\left(v_{12}, v_{13}\right)$, then $\mathcal{A}$ chooses the other edge. $\mathcal{B}$ has three choices: $\left(v_{12}, v_{14}\right),\left(v_{12}, v_{15}\right)$, or $\left(v_{14}, v_{15}\right)$.

- If $\mathcal{B}$ chooses $\left(v_{12}, v_{14}\right)$, then $\mathcal{A}$ chooses $\left(v_{13}, v_{15}\right)$. The resulting graph is in $\mathcal{L}_{1}(n-15)(b)$.

- If $\mathcal{B}$ chooses one of $\left(v_{12}, v_{15}\right)$ or $\left(v_{14}, v_{15}\right)$, then $\mathcal{A}$ chooses the other edge. The resulting graph is in $\mathcal{L}_{1}(n-15)(d)$.

- If $\mathcal{B}$ chooses $\left(v_{12}, v_{14}\right)$, then $\mathcal{A}$ chooses $\left(v_{11}, v_{14}\right)$. $\mathcal{B}$ has three choices: $\left(v_{12}, v_{13}\right)$, $\left(v_{12}, v_{15}\right)$, or $\left(v_{13}, v_{15}\right)$.

- If $\mathcal{B}$ chooses $\left(v_{12}, v_{13}\right)$, then $\mathcal{A}$ chooses $\left(v_{13}, v_{15}\right)$. The resulting graph is in $\mathcal{L}_{1}(n-15)(b)$.

- If $\mathcal{B}$ chooses one of $\left(v_{12}, v_{15}\right)$ or $\left(v_{13}, v_{15}\right)$, then $\mathcal{A}$ chooses the other edge. The resulting graph is in $\mathcal{L}_{1}(n-15)(d)$.

Case 3.3: $G_{n, 15}=G_{(3)}^{3}$. $\mathcal{B}$ has five choices: $\left(v_{8}, v_{10}\right),\left(v_{8}, v_{13}\right),\left(v_{10}, v_{12}\right),\left(v_{10}, v_{13}\right)$, or $\left(v_{12}, v_{13}\right)$.

Subcase 3.3.1: If $\mathcal{B}$ chooses one of $\left(v_{8}, v_{10}\right)$ or $\left(v_{10}, v_{13}\right)$, then $\mathcal{A}$ chooses the other edge. $\mathcal{B}$ has five choices: $\left(v_{9}, v_{13}\right),\left(v_{9}, v_{14}\right),\left(v_{12}, v_{13}\right),\left(v_{12}, v_{14}\right)$, or $\left(v_{13}, v_{14}\right)$.

- If $\mathcal{B}$ chooses one of $\left(v_{9}, v_{13}\right)$ or $\left(v_{13}, v_{14}\right)$, then $\mathcal{A}$ chooses the other edge. $\mathcal{B}$ has two choices: $\left(v_{12}, v_{14}\right)$ or $\left(v_{12}, v_{15}\right)$. $\mathcal{A}$ chooses whichever edge $\mathcal{B}$ did not. The resulting graph is in $\mathcal{L}_{1}(n-15)(a)$.

- If $\mathcal{B}$ chooses one of $\left(v_{9}, v_{14}\right)$ or $\left(v_{12}, v_{13}\right)$, then $\mathcal{A}$ chooses the other edge. $\mathcal{B}$ has three choices: $\left(v_{12}, v_{14}\right),\left(v_{12}, v_{15}\right)$, or $\left(v_{14}, v_{15}\right)$.

- If $\mathcal{B}$ chooses $\left(v_{12}, v_{14}\right)$, then $\mathcal{A}$ chooses $\left(v_{13}, v_{15}\right)$. The resulting graph is in $\mathcal{L}_{1}(n-15)(b)$. 
- If $\mathcal{B}$ chooses one of $\left(v_{12}, v_{15}\right)$ or $\left(v_{14}, v_{15}\right)$, then $\mathcal{A}$ chooses the other edge. The resulting graph is in $\mathcal{L}_{1}(n-15)(d)$.

- If $\mathcal{B}$ chooses $\left(v_{12}, v_{14}\right)$, then $\mathcal{A}$ chooses $\left(v_{9}, v_{14}\right)$. $\mathcal{B}$ has three choices: $\left(v_{12}, v_{13}\right)$, $\left(v_{12}, v_{15}\right)$, or $\left(v_{13}, v_{15}\right)$.

- If $\mathcal{B}$ chooses $\left(v_{12}, v_{13}\right)$, then $\mathcal{A}$ chooses $\left(v_{13}, v_{15}\right)$. The resulting graph is in $\mathcal{L}_{1}(n-15)(b)$.

- If $\mathcal{B}$ chooses one of $\left(v_{12}, v_{15}\right)$ or $\left(v_{13}, v_{15}\right)$, then $\mathcal{A}$ chooses the other edge. The resulting graph is in $\mathcal{L}_{1}(n-15)(d)$.

Subcase 3.3.2: If $\mathcal{B}$ chooses $\left(v_{8}, v_{13}\right)$, then $\mathcal{A}$ chooses $\left(v_{9}, v_{10}\right)$. $\mathcal{B}$ has five choices: $\left(v_{10}, v_{12}\right),\left(v_{10}, v_{14}\right),\left(v_{12}, v_{13}\right)$, or $\left(v_{12}, v_{14}\right)$.

- If $\mathcal{B}$ chooses one of $\left(v_{10}, v_{12}\right)$ or $\left(v_{12}, v_{14}\right)$, then $\mathcal{A}$ chooses the other edge. $\mathcal{B}$ has two choices: $\left(v_{13}, v_{14}\right)$ or $\left(v_{13}, v_{15}\right)$. $\mathcal{A}$ chooses whichever edge $\mathcal{B}$ did not. The resulting graph is in $\mathcal{L}_{1}(n-15)(a)$.

- If $\mathcal{B}$ chooses one of $\left(v_{10}, v_{14}\right)$ or $\left(v_{12}, v_{13}\right)$, then $\mathcal{A}$ chooses the other edge. $\mathcal{B}$ has three choices: $\left(v_{12}, v_{14}\right),\left(v_{12}, v_{15}\right)$, or $\left(v_{14}, v_{15}\right)$.

If $\mathcal{B}$ chooses $\left(v_{12}, v_{14}\right)$, then $\mathcal{A}$ chooses $\left(v_{13}, v_{15}\right)$.

If $\mathcal{B}$ chooses $\left(v_{12}, v_{15}\right)$, then $\mathcal{A}$ chooses $\left(v_{13}, v_{14}\right)$.

If $\mathcal{B}$ chooses $\left(v_{14}, v_{15}\right)$, then $\mathcal{A}$ chooses $\left(v_{12}, v_{14}\right)$.

The resulting three graphs are in $\mathcal{L}_{1}(n-15)(b)$.

Subcase 3.3.3: If $\mathcal{B}$ chooses one of $\left(v_{10}, v_{12}\right)$ or $\left(v_{12}, v_{13}\right)$, then $\mathcal{A}$ chooses the other edge. $\mathcal{B}$ has five choices: $\left(v_{8}, v_{10}\right),\left(v_{8}, v_{13}\right),\left(v_{8}, v_{14}\right),\left(v_{10}, v_{14}\right)$, or $\left(v_{13}, v_{14}\right)$.

- If $\mathcal{B}$ chooses $\left(v_{8}, v_{10}\right)$, then $\mathcal{A}$ chooses $\left(v_{9}, v_{14}\right)$. $\mathcal{B}$ has two choices: $\left(v_{13}, v_{14}\right)$ or $\left(v_{13}, v_{15}\right)$. $\mathcal{A}$ chooses whichever edge $\mathcal{B}$ did not. The resulting graph is in $\mathcal{L}_{1}(n-$ $15)(a)$.

- If $\mathcal{B}$ chooses $\left(v_{8}, v_{13}\right)$, then $\mathcal{A}$ chooses $\left(v_{9}, v_{14}\right) . \mathcal{B}$ has three choices: $\left(v_{10}, v_{14}\right)$, $\left(v_{10}, v_{15}\right)$, or $\left(v_{14}, v_{15}\right)$.

If $\mathcal{B}$ chooses $\left(v_{10}, v_{14}\right)$, then $\mathcal{A}$ chooses $\left(v_{13}, v_{15}\right)$.

If $\mathcal{B}$ chooses $\left(v_{10}, v_{15}\right)$, then $\mathcal{A}$ chooses $\left(v_{13}, v_{14}\right)$.

If $\mathcal{B}$ chooses $\left(v_{14}, v_{15}\right)$, then $\mathcal{A}$ chooses $\left(v_{10}, v_{14}\right)$.

The resulting three graphs are in $\mathcal{L}_{1}(n-15)(b)$.

- If $\mathcal{B}$ chooses $\left(v_{8}, v_{14}\right)$, then $\mathcal{A}$ chooses $\left(v_{9}, v_{10}\right)$. $\mathcal{B}$ has two choices: $\left(v_{13}, v_{14}\right)$ or $\left(v_{13}, v_{15}\right)$. $\mathcal{A}$ chooses whichever edge $\mathcal{B}$ did not. The resulting graph is in $\mathcal{L}_{1}(n-$ $15)(a)$. 
- If $\mathcal{B}$ chooses one of $\left(v_{10}, v_{14}\right)$ or $\left(v_{13}, v_{14}\right)$, then $\mathcal{A}$ chooses the other edge. $\mathcal{B}$ has three choices: $\left(v_{8}, v_{13}\right),\left(v_{8}, v_{15}\right)$, or $\left(v_{13}, v_{15}\right)$.

If $\mathcal{B}$ chooses $\left(v_{8}, v_{13}\right)$, then $\mathcal{A}$ chooses $\left(v_{9}, v_{15}\right)$.

If $\mathcal{B}$ chooses $\left(v_{8}, v_{15}\right)$, then $\mathcal{A}$ chooses $\left(v_{9}, v_{13}\right)$.

If $\mathcal{B}$ chooses $\left(v_{13}, v_{15}\right)$, then $\mathcal{A}$ chooses $\left(v_{8}, v_{14}\right)$.

The resulting three graphs are in $\mathcal{L}_{1}(n-15)(b)$.

Lemma 5.2. In $\Gamma_{\{B 3, T, C\}}(n), \mathcal{B}$ can choose edges $e_{2}, e_{4}, \ldots, e_{k}$ so that:

- when $n \geq 9$ and $k=12, G_{n, 12} \in \mathcal{L}_{1}(n-9)$, or

- when $n \geq 13$ and $k=18, G_{n, 18} \in \mathcal{L}_{1}(n-13)$.

Proof. Let $n \geq 9$. Up to isomorphism, $\mathcal{A}$ must choose $\left(v_{1}, v_{2}\right)$. $\mathcal{B}$ chooses $\left(v_{1}, v_{3}\right)$. $\mathcal{A}$ has two choices: $\left(v_{1}, v_{4}\right)$ or $\left(v_{2}, v_{4}\right)$.

- If $\mathcal{A}$ chooses $\left(v_{1}, v_{4}\right)$, then $\mathcal{B}$ chooses $\left(v_{2}, v_{5}\right)$.

- If $\mathcal{A}$ chooses $\left(v_{2}, v_{4}\right)$, then $\mathcal{B}$ chooses $\left(v_{1}, v_{5}\right)$.

The two resulting graphs are isomorphic, so without loss of generality we may consider the first case. $\mathcal{A}$ has four choices: $\left(v_{2}, v_{6}\right),\left(v_{3}, v_{5}\right),\left(v_{3}, v_{6}\right)$, or $\left(v_{5}, v_{6}\right)$.

- If $\mathcal{A}$ chooses one of $\left(v_{2}, v_{6}\right)$ or $\left(v_{3}, v_{5}\right)$, then $\mathcal{B}$ chooses the other edge.

If $\mathcal{A}$ chooses $\left(v_{3}, v_{6}\right)$, then $\mathcal{B}$ chooses $\left(v_{3}, v_{5}\right)$.

The two resulting graphs are isomorphic, so without loss of generality, we may consider the first case. $\mathcal{A}$ has four choices: $\left(v_{3}, v_{6}\right),\left(v_{3}, v_{7}\right),\left(v_{4}, v_{6}\right)$, or $\left(v_{4}, v_{7}\right)$.

- If $\mathcal{A}$ chooses $\left(v_{3}, v_{6}\right)$, then $\mathcal{B}$ chooses $\left(v_{5}, v_{7}\right)$. Call this graph $G_{(1)}$.

- If $\mathcal{A}$ chooses one of $\left(v_{3}, v_{7}\right)$ or $\left(v_{4}, v_{6}\right)$, then $\mathcal{B}$ chooses the other edge. Call this graph $G_{(2)}$.

- If $\mathcal{A}$ chooses $\left(v_{4}, v_{7}\right)$, then $\mathcal{B}$ chooses $\left(v_{3}, v_{7}\right)$. This graph is isomorphic to $G_{(2)}$.

- If $\mathcal{A}$ chooses $\left(v_{5}, v_{6}\right)$, then $\mathcal{B}$ chooses $\left(v_{3}, v_{5}\right)$. $\mathcal{A}$ has three choices: $\left(v_{2}, v_{7}\right),\left(v_{4}, v_{6}\right)$, or $\left(v_{4}, v_{7}\right)$.

- If $\mathcal{A}$ chooses one of $\left(v_{2}, v_{7}\right)$ or $\left(v_{4}, v_{7}\right)$, then $\mathcal{B}$ chooses the other edge. This graph is isomorphic to $G_{(2)}$ above.

- If $\mathcal{A}$ chooses $\left(v_{4}, v_{6}\right)$, then $\mathcal{B}$ chooses $\left(v_{2}, v_{7}\right)$. Call this graph $G_{(3)}$.

We can now examine the three graphs $G_{(i)}$ for $i=1,2,3$.

Case 1: $G_{n, 8}=G_{(1)}$. $\mathcal{A}$ has four choices: $\left(v_{4}, v_{6}\right),\left(v_{4}, v_{7}\right),\left(v_{4}, v_{8}\right)$, or $\left(v_{6}, v_{8}\right)$. 
- If $\mathcal{A}$ chooses one of $\left(v_{4}, v_{6}\right)$ or $\left(v_{4}, v_{8}\right)$, then $\mathcal{B}$ chooses the other edge. $\mathcal{A}$ has two choices: $\left(v_{7}, v_{8}\right)$ or $\left(v_{7}, v_{9}\right)$. $\mathcal{B}$ chooses whichever edge $\mathcal{A}$ did not. The resulting graph is in $\mathcal{L}_{1}(n-11)(a)$.

- If $\mathcal{A}$ chooses one of $\left(v_{4}, v_{7}\right)$ or $\left(v_{6}, v_{8}\right)$, then $\mathcal{B}$ chooses the other edge. $\mathcal{A}$ has three choices: $\left(v_{4}, v_{8}\right),\left(v_{4}, v_{9}\right)$, or $\left(v_{8}, v_{9}\right)$.

If $\mathcal{A}$ chooses $\left(v_{4}, v_{8}\right), \mathcal{B}$ chooses $\left(v_{7}, v_{9}\right)$.

If $\mathcal{A}$ chooses $\left(v_{4}, v_{9}\right), \mathcal{B}$ chooses $\left(v_{7}, v_{8}\right)$.

If $\mathcal{A}$ chooses $\left(v_{8}, v_{9}\right), \mathcal{B}$ chooses $\left(v_{4}, v_{8}\right)$.

In each case, the resulting graph is in $\mathcal{L}_{1}(n-11)(b)$.

Case 2: $G_{n, 8}=G_{(2)}$. $\mathcal{A}$ has seven choices: $\left(v_{4}, v_{5}\right),\left(v_{4}, v_{7}\right),\left(v_{4}, v_{8}\right),\left(v_{5}, v_{8}\right),\left(v_{6}, v_{7}\right)$, $\left(v_{6}, v_{8}\right)$, or $\left(v_{7}, v_{8}\right)$.

- If $\mathcal{A}$ chooses one of $\left(v_{4}, v_{5}\right)$ or $\left(v_{6}, v_{8}\right)$, then $\mathcal{B}$ chooses the other edge. $\mathcal{A}$ has two choices: $\left(v_{7}, v_{8}\right)$ or $\left(v_{7}, v_{9}\right)$. $\mathcal{B}$ chooses whichever edge $\mathcal{A}$ did not. The resulting graph is in $\mathcal{L}_{1}(n-11)(a)$.

- If $\mathcal{A}$ chooses one of $\left(v_{4}, v_{7}\right)$ or $\left(v_{5}, v_{8}\right)$, then $\mathcal{B}$ chooses the other edge. $\mathcal{A}$ has three choices: $\left(v_{6}, v_{8}\right),\left(v_{6}, v_{9}\right)$, or $\left(v_{8}, v_{9}\right)$.

If $\mathcal{A}$ chooses $\left(v_{6}, v_{8}\right), \mathcal{B}$ chooses $\left(v_{7}, v_{9}\right)$.

If $\mathcal{A}$ chooses $\left(v_{6}, v_{9}\right), \mathcal{B}$ chooses $\left(v_{7}, v_{8}\right)$.

If $\mathcal{A}$ chooses $\left(v_{8}, v_{9}\right), \mathcal{B}$ chooses $\left(v_{6}, v_{8}\right)$.

In each case, the resulting graph is in $\mathcal{L}_{1}(n-11)(b)$.

- If $\mathcal{A}$ chooses $\left(v_{4}, v_{8}\right)$, then $\mathcal{B}$ chooses $\left(v_{5}, v_{8}\right)$. $\mathcal{A}$ has three choices: $\left(v_{6}, v_{7}\right),\left(v_{6}, v_{9}\right)$, or $\left(v_{7}, v_{9}\right)$.

If $\mathcal{A}$ chooses $\left(v_{6}, v_{7}\right), \mathcal{B}$ chooses $\left(v_{7}, v_{9}\right)$.

If $\mathcal{A}$ chooses $\left(v_{6}, v_{9}\right), \mathcal{B}$ chooses $\left(v_{7}, v_{8}\right)$.

If $\mathcal{A}$ chooses $\left(v_{7}, v_{9}\right), \mathcal{B}$ chooses $\left(v_{6}, v_{7}\right)$.

In each case, the resulting graph is in $\mathcal{L}_{1}(n-11)(b)$.

- If $\mathcal{A}$ chooses $\left(v_{6}, v_{7}\right)$, then $\mathcal{B}$ chooses $\left(v_{4}, v_{8}\right)$. $\mathcal{A}$ has three choices: $\left(v_{5}, v_{8}\right),\left(v_{5}, v_{9}\right)$, or $\left(v_{8}, v_{9}\right)$.

If $\mathcal{A}$ chooses $\left(v_{5}, v_{8}\right), \mathcal{B}$ chooses $\left(v_{7}, v_{9}\right)$.

If $\mathcal{A}$ chooses $\left(v_{5}, v_{9}\right), \mathcal{B}$ chooses $\left(v_{7}, v_{8}\right)$.

If $\mathcal{A}$ chooses $\left(v_{8}, v_{9}\right), \mathcal{B}$ chooses $\left(v_{5}, v_{8}\right)$.

In each case, the resulting graph is in $\mathcal{L}_{1}(n-11)(b)$. 
- If $\mathcal{A}$ chooses $\left(v_{7}, v_{8}\right)$, then $\mathcal{B}$ chooses $\left(v_{4}, v_{7}\right) . \mathcal{A}$ has three choices: $\left(v_{5}, v_{8}\right),\left(v_{5}, v_{9}\right)$, or $\left(v_{8}, v_{9}\right)$.

If $\mathcal{A}$ chooses $\left(v_{5}, v_{8}\right), \mathcal{B}$ chooses $\left(v_{6}, v_{9}\right)$.

If $\mathcal{A}$ chooses $\left(v_{5}, v_{9}\right), \mathcal{B}$ chooses $\left(v_{6}, v_{8}\right)$.

If $\mathcal{A}$ chooses $\left(v_{8}, v_{9}\right), \mathcal{B}$ chooses $\left(v_{5}, v_{8}\right)$.

In each case, the resulting graph is in $\mathcal{L}_{1}(n-11)(b)$.

Case 3: $G_{n, 8}=G_{(3)}$. $\mathcal{A}$ has five choices: $\left(v_{3}, v_{7}\right),\left(v_{3}, v_{8}\right),\left(v_{4}, v_{7}\right),\left(v_{4}, v_{8}\right)$, or $\left(v_{7}, v_{8}\right)$.

- If $\mathcal{A}$ chooses one of $\left(v_{3}, v_{7}\right)$ or $\left(v_{7}, v_{8}\right)$, then $\mathcal{B}$ chooses the other edge. $\mathcal{A}$ has three choices: $\left(v_{4}, v_{8}\right),\left(v_{4}, v_{9}\right)$, or $\left(v_{8}, v_{9}\right)$.

If $\mathcal{A}$ chooses $\left(v_{4}, v_{8}\right), \mathcal{B}$ chooses $\left(v_{6}, v_{9}\right)$.

If $\mathcal{A}$ chooses $\left(v_{4}, v_{9}\right), \mathcal{B}$ chooses $\left(v_{6}, v_{8}\right)$.

If $\mathcal{A}$ chooses $\left(v_{8}, v_{9}\right), \mathcal{B}$ chooses $\left(v_{4}, v_{8}\right)$.

In each case, the resulting graph is in $\mathcal{L}_{1}(n-11)(b)$.

- If $\mathcal{A}$ chooses one of $\left(v_{3}, v_{8}\right)$ or $\left(v_{4}, v_{7}\right)$, then $\mathcal{B}$ chooses the other edge. $\mathcal{A}$ has three choices: $\left(v_{6}, v_{8}\right),\left(v_{6}, v_{9}\right)$, or $\left(v_{8}, v_{9}\right)$.

If $\mathcal{A}$ chooses $\left(v_{6}, v_{8}\right), \mathcal{B}$ chooses $\left(v_{7}, v_{9}\right)$.

If $\mathcal{A}$ chooses $\left(v_{6}, v_{9}\right), \mathcal{B}$ chooses $\left(v_{7}, v_{8}\right)$.

If $\mathcal{A}$ chooses $\left(v_{8}, v_{9}\right), \mathcal{B}$ chooses $\left(v_{6}, v_{8}\right)$.

In each case, the resulting graph is in $\mathcal{L}_{1}(n-11)(b)$.

- If $\mathcal{A}$ chooses one of $\left(v_{4}, v_{8}\right)$, then $\mathcal{B}$ chooses $\left(v_{3}, v_{8}\right)$. $\mathcal{A}$ has three choices: $\left(v_{6}, v_{7}\right)$, $\left(v_{6}, v_{9}\right)$, or $\left(v_{7}, v_{9}\right)$.

If $\mathcal{A}$ chooses $\left(v_{6}, v_{7}\right), \mathcal{B}$ chooses $\left(v_{7}, v_{9}\right)$.

If $\mathcal{A}$ chooses $\left(v_{6}, v_{9}\right), \mathcal{B}$ chooses $\left(v_{7}, v_{8}\right)$.

If $\mathcal{A}$ chooses $\left(v_{7}, v_{9}\right), \mathcal{B}$ chooses $\left(v_{6}, v_{7}\right)$.

In each case, the resulting graph is in $\mathcal{L}_{1}(n-11)(b)$.

Now let $n \geq 13$. Up to isomorphism, $\mathcal{A}$ must choose $\left(v_{1}, v_{2}\right)$. $\mathcal{B}$ chooses $\left(v_{1}, v_{3}\right)$. $\mathcal{A}$ has two choices: $\left(v_{1}, v_{4}\right)$ or $\left(v_{2}, v_{4}\right)$.

- If $\mathcal{A}$ chooses $\left(v_{1}, v_{4}\right)$, then $\mathcal{B}$ chooses $\left(v_{2}, v_{5}\right)$.

- If $\mathcal{A}$ chooses $\left(v_{2}, v_{4}\right)$, then $\mathcal{B}$ chooses $\left(v_{1}, v_{5}\right)$.

The two resulting graphs are isomorphic, so without loss of generality we may assume the first case. $\mathcal{A}$ has four choices: $\left(v_{2}, v_{6}\right),\left(v_{3}, v_{5}\right),\left(v_{3}, v_{6}\right)$, or $\left(v_{5}, v_{6}\right)$. 
- If $\mathcal{A}$ chooses $\left(v_{2}, v_{6}\right)$, then $\mathcal{B}$ chooses $\left(v_{3}, v_{7}\right)$.

If $\mathcal{A}$ chooses $\left(v_{3}, v_{6}\right)$, then $\mathcal{B}$ chooses $\left(v_{2}, v_{7}\right)$.

If $\mathcal{A}$ chooses $\left(v_{5}, v_{6}\right)$, then $\mathcal{B}$ chooses $\left(v_{2}, v_{7}\right)$.

The three resulting graphs are isomorphic, so without loss of generality we may assume the first case. $\mathcal{A}$ has eight choices: $\left(v_{3}, v_{5}\right),\left(v_{3}, v_{8}\right),\left(v_{4}, v_{5}\right),\left(v_{4}, v_{7}\right),\left(v_{4}, v_{8}\right)$, $\left(v_{5}, v_{7}\right),\left(v_{5}, v_{8}\right)$, or $\left(v_{7}, v_{8}\right)$.

- If $\mathcal{A}$ chooses one of $\left(v_{3}, v_{5}\right)$ or $\left(v_{4}, v_{5}\right)$, then $\mathcal{B}$ chooses the other edge. Call this graph $G_{(1)}^{1}$.

- If $\mathcal{A}$ chooses one of $\left(v_{3}, v_{8}\right)$ or $\left(v_{5}, v_{7}\right)$, then $\mathcal{B}$ chooses the other edge. Call this graph $G_{(2)}^{1}$.

- If $\mathcal{A}$ chooses $\left(v_{4}, v_{7}\right)$, then $\mathcal{B}$ chooses $\left(v_{5}, v_{7}\right)$. Call this graph $G_{(3)}^{1}$.

- If $\mathcal{A}$ chooses one of $\left(v_{4}, v_{8}\right)$ or $\left(v_{7}, v_{8}\right)$, then $\mathcal{B}$ chooses the other edge. Call this graph $G_{(4)}^{1}$.

- If $\mathcal{A}$ chooses $\left(v_{5}, v_{8}\right)$, then $\mathcal{B}$ chooses $\left(v_{5}, v_{7}\right)$. This graph is isomorphic to $G_{(2)}^{1}$.

- If $\mathcal{A}$ chooses $\left(v_{3}, v_{5}\right)$, then $\mathcal{B}$ chooses $\left(v_{4}, v_{5}\right)$. $\mathcal{A}$ must choose $\left(v_{2}, v_{6}\right)$. Then $\mathcal{B}$ chooses $\left(v_{3}, v_{7}\right)$. This graph is isomorphic to $G_{(1)}^{1}$.

Now we need to consider the four graphs $G_{(i)}^{1}$ for $i=1,2,3,4$.

Case 1.1: $G_{n, 8}=G_{(1)}^{1}$. $\mathcal{A}$ has four choices: $\left(v_{4}, v_{6}\right),\left(v_{4}, v_{8}\right),\left(v_{6}, v_{7}\right)$, or $\left(v_{6}, v_{8}\right)$.

- If $\mathcal{A}$ chooses one of $\left(v_{4}, v_{6}\right)$ or $\left(v_{6}, v_{7}\right)$, then $\mathcal{B}$ the other edge. Call this graph $G_{(1)}^{2}$.

- If $\mathcal{A}$ chooses $\left(v_{4}, v_{8}\right)$, then $\mathcal{B}$ chooses $\left(v_{6}, v_{9}\right)$.

If $\mathcal{A}$ chooses $\left(v_{6}, v_{8}\right)$, then $\mathcal{B}$ chooses $\left(v_{4}, v_{9}\right)$.

The two resulting graphs are isomorphic, so without loss of generality we may consider the first case. Call this graph $G_{(2)}^{2}$.

Case 1.2: $G_{n, 8}=G_{(2)}^{1}$. $\mathcal{A}$ has seven choices:

- If $\mathcal{A}$ chooses $\left(v_{4}, v_{5}\right)$, then $\mathcal{B}$ chooses $\left(v_{7}, v_{9}\right)$.

If $\mathcal{A}$ chooses $\left(v_{5}, v_{8}\right)$, then $\mathcal{B}$ chooses $\left(v_{7}, v_{9}\right)$.

If $\mathcal{A}$ chooses $\left(v_{6}, v_{9}\right)$, then $\mathcal{B}$ chooses $\left(v_{6}, v_{7}\right)$.

The three resulting graphs are isomorphic, so we may consider the first case. Call this graph $G_{(3)}^{2}$.

- If $\mathcal{A}$ chooses $\left(v_{4}, v_{6}\right)$, then $\mathcal{B}$ chooses $\left(v_{7}, v_{9}\right)$.

If $\mathcal{A}$ chooses one of $\left(v_{4}, v_{9}\right)$ or $\left(v_{5}, v_{9}\right)$, then $\mathcal{B}$ chooses the other edge.

If $\mathcal{A}$ chooses $\left(v_{6}, v_{8}\right)$, then $\mathcal{B}$ chooses $\left(v_{5}, v_{9}\right)$.

The three resulting graphs satisfy: 
1. $(z(G), p(G), t(G))=(n-9,2,3)$,

2. if $P(G)=\left\{u_{1}, u_{2}\right\}$ and $T(G)=\left\{u_{3}, u_{4}, u_{5}\right\}$, then, up to isomorphism, $\left(u_{3}, u_{4}\right) \in E(G),\left(u_{1}, u_{5}\right),\left(u_{4}, u_{5}\right) \in F(G)$, and all other pairs are in $(E(G) \cup$ $F(G))^{c}$.

Gameplay is identical in each case, so we may consider the first case. Call this graph $G_{(4)}^{2}$.

Case 1.3: $G_{n, 8}=G_{(3)}^{1}$. $\mathcal{A}$ has four choices: $\left(v_{3}, v_{6}\right),\left(v_{3}, v_{8}\right),\left(v_{5}, v_{8}\right)$, or $\left(v_{6}, v_{8}\right)$.

- If $\mathcal{A}$ chooses $\left(v_{3}, v_{6}\right)$, then $\mathcal{B}$ chooses $\left(v_{4}, v_{6}\right)$. This graph is isomorphic to $G_{(1)}^{2}$.

- If $\mathcal{A}$ chooses $\left(v_{3}, v_{8}\right)$, then $\mathcal{B}$ chooses $\left(v_{5}, v_{9}\right)$. This graph is isomorphic to $G_{(3)}^{2}$.

- If $\mathcal{A}$ chooses $\left(v_{5}, v_{8}\right)$, then $\mathcal{B}$ chooses $\left(v_{3}, v_{9}\right)$. This graph is isomorphic to $G_{(3)}^{2}$.

- If $\mathcal{A}$ chooses $\left(v_{6}, v_{8}\right)$, then $\mathcal{B}$ chooses $\left(v_{6}, v_{9}\right)$. Call this graph $G_{(5)}^{2}$.

Case 1.4: $G_{n, 8}=G_{(4)}^{1}$. $\mathcal{A}$ has five choices: $\left(v_{3}, v_{5}\right),\left(v_{3}, v_{9}\right),\left(v_{5}, v_{7}\right),\left(v_{5}, v_{9}\right)$, or $\left(v_{7}, v_{9}\right)$.

- If $\mathcal{A}$ chooses one of $\left(v_{3}, v_{5}\right)$ or $\left(v_{5}, v_{9}\right)$, then $\mathcal{B}$ chooses the other edge. Call this graph $G_{(6)}^{2}$.

- If $\mathcal{A}$ chooses $\left(v_{3}, v_{9}\right)$, then $\mathcal{B}$ chooses $\left(v_{8}, v_{9}\right)$. This graph is isomorphic to $G_{(5)}^{2}$.

- If $\mathcal{A}$ chooses $\left(v_{5}, v_{7}\right)$, then $\mathcal{B}$ chooses $\left(v_{3}, v_{9}\right)$. This graph satisfies:

1. $(z(G), p(G), t(G))=(n-9,2,3)$,

2. if $P(G)=\left\{u_{1}, u_{2}\right\}$ and $T(G)=\left\{u_{3}, u_{4}, u_{5}\right\}$, then, up to isomorphism, $\left(u_{3}, u_{4}\right) \in E(G),\left(u_{1}, u_{5}\right),\left(u_{4}, u_{5}\right) \in F(G)$, and all other pairs are in $(E(G) \cup$ $F(G))^{c}$.

Gameplay is identical to that of $G_{(4)}^{2}$.

- If $\mathcal{A}$ chooses $\left(v_{7}, v_{9}\right)$, then $\mathcal{B}$ chooses $\left(v_{4}, v_{9}\right)$. This graph is isomorphic to $G_{(5)}^{2}$.

We now need to look at the graphs $G_{(i)}^{2}$ for $1 \leq i \leq 6$. $G_{(1)}^{3}$

Case 2.1: $G_{n, 10}=G_{(1)}^{2}$. $\mathcal{A}$ must choose $\left(v_{7}, v_{8}\right)$. $\mathcal{B}$ chooses $\left(v_{8}, v_{9}\right)$. Call this graph

Case 2.2: $G_{n, 10}=G_{(2)}^{2}$. $\mathcal{A}$ has six choices: $\left(v_{6}, v_{7}\right),\left(v_{6}, v_{10}\right),\left(v_{7}, v_{8}\right),\left(v_{7}, v_{9}\right),\left(v_{7}, v_{10}\right)$, or $\left(v_{9}, v_{10}\right)$.

- If $\mathcal{A}$ chooses one of $\left(v_{6}, v_{7}\right)$ or $\left(v_{7}, v_{10}\right)$, then $\mathcal{B}$ chooses the other edge. Call this $\operatorname{graph} G_{(2)}^{3}$. 
- If $\mathcal{A}$ chooses one of $\left(v_{6}, v_{10}\right)$ or $\left(v_{7}, v_{8}\right)$, then $\mathcal{B}$ chooses the other edge. Call this $\operatorname{graph} G_{(3)}^{3}$.

- If $\mathcal{A}$ chooses one of $\left(v_{7}, v_{9}\right)$ or $\left(v_{9}, v_{10}\right)$, then $\mathcal{B}$ chooses the other edge. Call this $\operatorname{graph} G_{(4)}^{3}$.

Case 2.3: $G_{n, 10}=G_{(3)}^{2}$. $\mathcal{A}$ has four choices: $\left(v_{4}, v_{6}\right),\left(v_{4}, v_{10}\right),\left(v_{6}, v_{8}\right)$, or $\left(v_{6}, v_{10}\right)$.

- If $\mathcal{A}$ chooses one of $\left(v_{4}, v_{6}\right)$ or $\left(v_{6}, v_{10}\right)$, then $\mathcal{B}$ chooses the other edge. This graph satisfies:

1. $(z(G), p(G), t(G))=(n-10,3,0)$, and

2. for each pair $u_{1}, u_{2} \in P(G),\left(u_{1}, u_{2}\right) \notin(E(G) \cup F(G))$.

Gameplay is identical to that of $G_{(2)}^{3}$.

- If $\mathcal{A}$ chooses one of $\left(v_{4}, v_{10}\right)$ or $\left(v_{6}, v_{8}\right)$, then $\mathcal{B}$ chooses the other edge. Call this $\operatorname{graph} G_{(5)}^{3}$.

Case 2.4: $G_{n, 10}=G_{(4)}^{2}$. $\mathcal{A}$ has twelve choices: $\left(v_{4}, v_{5}\right),\left(v_{4}, v_{8}\right),\left(v_{4}, v_{9}\right),\left(v_{4}, v_{10}\right)$, $\left(v_{5}, v_{8}\right),\left(v_{5}, v_{10}\right),\left(v_{6}, v_{8}\right),\left(v_{6}, v_{9}\right),\left(v_{6}, v_{10}\right),\left(v_{8}, v_{9}\right),\left(v_{8}, v_{10}\right)$, or $\left(v_{9}, v_{10}\right)$.

- If $\mathcal{A}$ chooses one of $\left(v_{4}, v_{5}\right)$ or $\left(v_{6}, v_{10}\right)$, then $\mathcal{B}$ chooses the other edge. This graph satisfies:

1. $(z(G), p(G), t(G))=(n-10,3,0)$, and

2. for each pair $u_{1}, u_{2} \in P(G),\left(u_{1}, u_{2}\right) \notin(E(G) \cup F(G))$.

Gameplay is identical to that of $G_{(2)}^{3}$.

- If $\mathcal{A}$ chooses one of $\left(v_{4}, v_{8}\right)$ or $\left(v_{5}, v_{10}\right)$, then $\mathcal{B}$ chooses the other edge.

If $\mathcal{A}$ chooses $\left(v_{4}, v_{9}\right)$, then $\mathcal{B}$ chooses $\left(v_{5}, v_{10}\right)$.

If $\mathcal{A}$ chooses $\left(v_{4}, v_{10}\right)$, then $\mathcal{B}$ chooses $\left(v_{5}, v_{10}\right)$.

If $\mathcal{A}$ chooses $\left(v_{6}, v_{8}\right)$, then $\mathcal{B}$ chooses $\left(v_{5}, v_{10}\right)$.

If $\mathcal{A}$ chooses $\left(v_{6}, v_{9}\right)$, then $\mathcal{B}$ chooses $\left(v_{4}, v_{10}\right)$.

In each case, the resulting graph satisfies:

1. $(z(G), p(G), t(G))=(n-10,2,2)$,

2. if $T(G)=\left\{u_{1}, u_{2}\right\}$, then $\left(u_{1}, u_{2}\right) \in F(G)$, and

3. all other pairs are in $(E(G) \cup F(G))^{c}$.

Gameplay is identical in each case, so we may consider the first one. Call this graph $G_{(6)}^{3}$. 
- If $\mathcal{A}$ chooses one of $\left(v_{5}, v_{8}\right)$ or $\left(v_{8}, v_{10}\right)$, then $\mathcal{B}$ chooses the other edge. This graph satisfies:

1. $(z(G), p(G), t(G))=(n-10,2,2)$,

2. if $T(G)=\left\{u_{1}, u_{2}\right\}$, then $\left(u_{1}, u_{2}\right) \in E(G)$, and

3. all other pairs are in $(E(G) \cup F(G))^{c}$.

Gameplay is identical to that of $G_{(5)}^{3}$.

- If $\mathcal{A}$ chooses $\left(v_{8}, v_{9}\right)$, then $\mathcal{B}$ chooses or $\left(v_{5}, v_{10}\right)$.

If $\mathcal{A}$ chooses $\left(v_{9}, v_{10}\right)$, then $\mathcal{B}$ chooses or $\left(v_{5}, v_{10}\right)$.

These two graphs are isomorphic, so we may consider the first case. Call this graph $G_{(7)}^{3}$.

Case 2.5: $G_{n, 10}=G_{(5)}^{2}$. $\mathcal{A}$ has three choices: $\left(v_{3}, v_{8}\right),\left(v_{3}, v_{10}\right)$, or $\left(v_{8}, v_{10}\right)$.

- If $\mathcal{A}$ chooses one of $\left(v_{3}, v_{8}\right)$ or $\left(v_{8}, v_{10}\right)$, then $\mathcal{B}$ chooses the other edge. This graph satisfies:

1. $(z(G), p(G), t(G))=(n-10,2,2)$,

2. if $T(G)=\left\{u_{1}, u_{2}\right\}$, then $\left(u_{1}, u_{2}\right) \in F(G)$, and

3. all other pairs are in $(E(G) \cup F(G))^{c}$.

Gameplay is identical to that of $G_{(6)}^{3}$.

- If $\mathcal{A}$ chooses $\left(v_{3}, v_{10}\right)$, then $\mathcal{B}$ chooses $\left(v_{4}, v_{10}\right)$. Call this graph $G_{(8)}^{3}$.

Case 2.6: $G_{n, 10}=G_{(6)}^{2}$. $\mathcal{A}$ has six choices: $\left(v_{4}, v_{6}\right),\left(v_{4}, v_{10}\right),\left(v_{6}, v_{8}\right),\left(v_{6}, v_{9}\right),\left(v_{6}, v_{10}\right)$, or $\left(v_{8}, v_{10}\right)$.

- If $\mathcal{A}$ chooses one of $\left(v_{4}, v_{6}\right)$ or $\left(v_{6}, v_{10}\right)$, then $\mathcal{B}$ chooses the other edge. This graph satisfies:

1. $(z(G), p(G), t(G))=(n-10,2,2)$,

2. if $T(G)=\left\{u_{1}, u_{2}\right\}$, then $\left(u_{1}, u_{2}\right) \in E(G)$, and

3. all other pairs are in $(E(G) \cup F(G))^{c}$.

Gameplay is identical to that of $G_{(5)}^{3}$.

- If $\mathcal{A}$ chooses one of $\left(v_{4}, v_{10}\right)$ or $\left(v_{6}, v_{8}\right)$, then $\mathcal{B}$ chooses the other edge. This graph satisfies:

1. $(z(G), p(G), t(G))=(n-10,2,2)$, 
2. if $T(G)=\left\{u_{1}, u_{2}\right\}$, then $\left(u_{1}, u_{2}\right) \in F(G)$, and

3. all other pairs are in $(E(G) \cup F(G))^{c}$.

Gameplay is identical to that of $G_{(6)}^{3}$.

- If $\mathcal{A}$ chooses one of $\left(v_{6}, v_{9}\right)$ or $\left(v_{8}, v_{10}\right)$, then $\mathcal{B}$ chooses the other edge. Call this $\operatorname{graph} G_{(9)}^{3}$.

Now let us consider the graphs $G_{(i)}^{3}$ for $1 \leq i \leq 9$.

Case 3.1: $G_{n, 12}=G_{(1)}^{3}$. $\mathcal{A}$ has two choices: $\left(v_{8}, v_{10}\right)$ or $\left(v_{9}, v_{10}\right)$.

If $\mathcal{A}$ chooses $\left(v_{8}, v_{10}\right)$, then $\mathcal{B}$ chooses $\left(v_{9}, v_{11}\right)$.

If $\mathcal{A}$ chooses $\left(v_{8}, v_{10}\right)$, then $\mathcal{B}$ chooses $\left(v_{9}, v_{11}\right)$.

The two resulting graphs are isomorphic, so we may consider the first case. Call this graph $G_{(1)}^{4}$.

Case 3.2: $G_{n, 12}=G_{(2)}^{3}$. $\mathcal{A}$ has two choices: $\left(v_{8}, v_{9}\right)$ or $\left(v_{8}, v_{11}\right)$. $\mathcal{B}$ chooses whichever edge $\mathcal{A}$ did not. Call this graph $G_{(2)}^{4}$.

Case 3.3: $G_{n, 12}=G_{(3)}^{3}$. $\mathcal{A}$ has three choices: $\left(v_{7}, v_{9}\right),\left(v_{7}, v_{11}\right)$, or $\left(v_{9}, v_{11}\right)$.

- If $\mathcal{A}$ chooses one of $\left(v_{7}, v_{9}\right)$ or $\left(v_{9}, v_{11}\right)$, then $\mathcal{B}$ chooses the other edge. Call this $\operatorname{graph} G_{(3)}^{4}$.

- If $\mathcal{A}$ chooses $\left(v_{7}, v_{11}\right)$, then $\mathcal{B}$ chooses $\left(v_{8}, v_{9}\right)$. This graph satisfies:

1. $(z(G), p(G), t(G))=(n-11,2,1)$,

2. if $P(G)=\left\{u_{1}, u_{2}\right\}$ and $T(G)=\left\{u_{3}\right\}$, then, up to isomorphism, $\left(u_{1}, u_{3}\right) \in$ $F(G)$, and

3. all other pairs are in $(E(G) \cup F(G))^{c}$.

Gameplay is identical to that of $G_{(2)}^{4}$.

Case 3.4: $G_{n, 12}=G_{(4)}^{3}$. $\mathcal{A}$ has five choices: $\left(v_{6}, v_{8}\right),\left(v_{6}, v_{11}\right),\left(v_{8}, v_{10}\right),\left(v_{8}, v_{11}\right)$, or $\left(v_{10}, v_{11}\right)$.

- If $\mathcal{A}$ chooses one of $\left(v_{6}, v_{8}\right)$ or $\left(v_{8}, v_{11}\right)$, then $\mathcal{B}$ chooses the other edge. This graph satisfies:

1. $(z(G), p(G), t(G))=(n-11,2,1)$,

2. if $P(G)=\left\{u_{1}, u_{2}\right\}$ and $T(G)=\left\{u_{3}\right\}$, then, up to isomorphism, $\left(u_{1}, u_{3}\right) \in$ $F(G)$, and

3. all other pairs are in $(E(G) \cup F(G))^{c}$.

Gameplay is identical to that of $G_{(2)}^{4}$. 
- If $\mathcal{A}$ chooses $\left(v_{6}, v_{11}\right)$, then $\mathcal{B}$ chooses $\left(v_{7}, v_{8}\right)$. This graph satisfies:

1. $(z(G), p(G), t(G))=(n-11,2,1)$, and

2. if $P(G)=\left\{u_{1}, u_{2}\right\}$ and $T(G)=\left\{u_{3}\right\}$, then all pairs are in $(E(G) \cup F(G))^{c}$.

Gameplay is identical to that of $G_{(3)}^{4}$.

- If $\mathcal{A}$ chooses one of $\left(v_{8}, v_{10}\right)$ or $\left(v_{10}, v_{11}\right)$, then $\mathcal{B}$ chooses the other edge. Call this $\operatorname{graph} G_{(4)}^{4}$.

Case 3.5: $G_{n, 12}=G_{(5)}^{3}$. $\mathcal{A}$ has four choices: $\left(v_{6}, v_{9}\right),\left(v_{6}, v_{11}\right),\left(v_{9}, v_{10}\right)$, or $\left(v_{9}, v_{11}\right)$.

- If $\mathcal{A}$ chooses $\left(v_{6}, v_{9}\right)$, then $\mathcal{B}$ chooses $\left(v_{8}, v_{11}\right)$.

If $\mathcal{A}$ chooses $\left(v_{9}, v_{11}\right)$, then $\mathcal{B}$ chooses $\left(v_{6}, v_{9}\right)$.

In each case, the resulting graph satisfies:

1. $(z(G), p(G), t(G))=(n-11,2,1)$, and

2. if $P(G)=\left\{u_{1}, u_{2}\right\}$ and $T(G)=\left\{u_{3}\right\}$, then all pairs are in $(E(G) \cup F(G))^{c}$.

Gameplay is identical to that of $G_{(3)}^{4}$.

- If $\mathcal{A}$ chooses one of $\left(v_{6}, v_{11}\right)$ or $\left(v_{9}, v_{10}\right)$, then $\mathcal{B}$ chooses the other edge. Call this $\operatorname{graph} G_{(5)}^{4}$.

Case 3.6: $G_{n, 12}=G_{(6)}^{3}$. $\mathcal{A}$ has four choices: $\left(v_{6}, v_{9}\right),\left(v_{6}, v_{11}\right),\left(v_{9}, v_{10}\right)$, or $\left(v_{9}, v_{11}\right)$.

- If $\mathcal{A}$ chooses $\left(v_{6}, v_{9}\right)$, then $\mathcal{B}$ chooses $\left(v_{8}, v_{11}\right)$.

If $\mathcal{A}$ chooses $\left(v_{6}, v_{11}\right)$, then $\mathcal{B}$ chooses $\left(v_{8}, v_{9}\right)$.

In each case, the resulting graph satisfies:

1. $(z(G), p(G), t(G))=(n-11,2,1)$, and

2. if $P(G)=\left\{u_{1}, u_{2}\right\}$ and $T(G)=\left\{u_{3}\right\}$, then all pairs are in $(E(G) \cup F(G))^{c}$.

Gameplay is identical to that of $G_{(3)}^{4}$.

- If $\mathcal{A}$ chooses one of $\left(v_{9}, v_{10}\right)$ or $\left(v_{9}, v_{11}\right)$, then $\mathcal{B}$ chooses the other edge. This graph satisfies:

1. $(z(G), p(G), t(G))=(n-11,1,3)$,

2. if $P(G)=\left\{u_{1},\right\}$ and $T(G)=\left\{u_{2}, u_{3}, u_{4}\right\}$, then, up to isomorphism, $\left(u_{1}, u_{2}\right),\left(u_{3}, u_{4}\right) \in F(G)$, and

3. all other pairs are in $(E(G) \cup F(G))^{c}$. 
Gameplay is identical to that of $G_{(4)}^{4}$.

Case 3.7: $G_{n, 12}=G_{(7)}^{3}$. $\mathcal{A}$ has four choices: $\left(v_{4}, v_{8}\right),\left(v_{4}, v_{10}\right),\left(v_{4}, v_{11}\right)$, or $\left(v_{10}, v_{11}\right)$.

- If $\mathcal{A}$ chooses $\left(v_{4}, v_{8}\right)$, then $\mathcal{B}$ chooses $\left(v_{6}, v_{11}\right)$.

If $\mathcal{A}$ chooses $\left(v_{4}, v_{11}\right)$, then $\mathcal{B}$ chooses $\left(v_{6}, v_{8}\right)$.

In each case, the resulting graph satisfies:

1. $(z(G), p(G), t(G))=(n-11,2,1)$, and

2. if $P(G)=\left\{u_{1}, u_{2}\right\}$ and $T(G)=\left\{u_{3}\right\}$, then all pairs are in $(E(G) \cup F(G))^{c}$.

Gameplay is identical to that of $G_{(3)}^{4}$.

- If $\mathcal{A}$ chooses $\left(v_{4}, v_{11}\right)$, then $\mathcal{B}$ chooses $\left(v_{6}, v_{8}\right)$.

If $\mathcal{A}$ chooses $\left(v_{10}, v_{11}\right)$, then $\mathcal{B}$ chooses $\left(v_{4}, v_{10}\right)$.

In each case, the resulting graph satisfies:

1. $(z(G), p(G), t(G))=(n-11,1,3)$,

2. if $P(G)=\left\{u_{1}\right\}$ and $T(G)=\left\{u_{2}, u_{3}, u_{4}\right\}$, then, up to isomorphism, $\left(u_{2}, u_{3}\right) \in$ $E(G)$, and

3. all other pairs are in $(E(G) \cup F(G))^{c}$.

Gameplay is identical in each case, so we can consider the first case. Call this graph $G_{(6)}^{4}$.

Case 3.8: $G_{n, 12}=G_{(8)}^{3}$. $\mathcal{A}$ has four choices: $\left(v_{5}, v_{8}\right),\left(v_{5}, v_{10}\right),\left(v_{5}, v_{11}\right)$, or $\left(v_{8}, v_{11}\right)$.

- If $\mathcal{A}$ chooses one of $\left(v_{5}, v_{8}\right)$ or $\left(v_{8}, v_{11}\right)$, then $\mathcal{B}$ chooses the other edge. This graph satisfies:

1. $(z(G), p(G), t(G))=(n-11,2,1)$, and

2. if $P(G)=\left\{u_{1}, u_{2}\right\}$ and $T(G)=\left\{u_{3}\right\}$, then all pairs are in $(E(G) \cup F(G))^{c}$.

Gameplay is identical to that of $G_{(3)}^{4}$.

- If $\mathcal{A}$ chooses $\left(v_{5}, v_{10}\right)$, then $\mathcal{B}$ chooses $\left(v_{8}, v_{11}\right)$. This graph satisfies:

1. $(z(G), p(G), t(G))=(n-11,2,1)$,

2. if $P(G)=\left\{u_{1}, u_{2}\right\}$ and $T(G)=\left\{u_{3}\right\}$, then, up to isomorphism, $\left(u_{1}, u_{3}\right) \in$ $E(G),\left(u_{2}, u_{3}\right) \in F(G)$, and

3. $\left(u_{1}, u_{3}\right) \in(E(G) \cup F(G))^{c}$. 
Gameplay is identical to that of $G_{(1)}^{4}$.

- If $\mathcal{A}$ chooses $\left(v_{5}, v_{11}\right)$, then $\mathcal{B}$ chooses $\left(v_{8}, v_{10}\right)$. This graph satisfies:

1. $(z(G), p(G), t(G))=(n-11,2,1)$,

2. if $P(G)=\left\{u_{1}, u_{2}\right\}$ and $T(G)=\left\{u_{3}\right\}$, then, up to isomorphism, $\left(u_{1}, u_{3}\right) \in$ $F(G)$, and

3. all other pairs are in $(E(G) \cup F(G))^{c}$.

Gameplay is identical to that of $G_{(2)}^{4}$.

Case 3.9: $G_{n, 12}=G_{(9)}^{3} \cdot \mathcal{A}$ has five choices: $\left(v_{4}, v_{6}\right),\left(v_{4}, v_{11}\right),\left(v_{6}, v_{10}\right),\left(v_{6}, v_{11}\right)$, or $\left(v_{10}, v_{11}\right)$.

- If $\mathcal{A}$ chooses $\left(v_{4}, v_{6}\right)$, then $\mathcal{B}$ chooses $\left(v_{7}, v_{11}\right)$.

If $\mathcal{A}$ chooses $\left(v_{4}, v_{11}\right)$, then $\mathcal{B}$ chooses $\left(v_{6}, v_{7}\right)$.

In each case, the resulting graph satisfies:

1. $(z(G), p(G), t(G))=(n-11,2,1)$, and

2. if $P(G)=\left\{u_{1}, u_{2}\right\}$ and $T(G)=\left\{u_{3}\right\}$, then all pairs are in $(E(G) \cup F(G))^{c}$.

Gameplay is identical to that of $G_{(3)}^{4}$.

- If $\mathcal{A}$ chooses $\left(v_{6}, v_{10}\right)$, then $\mathcal{B}$ chooses $\left(v_{4}, v_{9}\right)$. Call this graph $G_{(7)}^{4}$.

- If $\mathcal{A}$ chooses one of $\left(v_{6}, v_{11}\right)$ or $\left(v_{10}, v_{11}\right)$, then $\mathcal{B}$ chooses the other edge. Call this $\operatorname{graph} G_{(8)}^{4}$.

Now we consider the graphs $G_{(i)}^{4}$ for $1 \leq i \leq 8$.

Case 4.1: $G_{n, 14}=G_{(1)}^{4}$. $\mathcal{A}$ has four choices: $\left(v_{9}, v_{12}\right),\left(v_{10}, v_{11}\right),\left(v_{10}, v_{12}\right)$, or $\left(v_{11}, v_{12}\right)$.

- If $\mathcal{A}$ chooses one of $\left(v_{9}, v_{12}\right)$ or $\left(v_{10}, v_{11}\right)$, then $\mathcal{B}$ chooses the other edge.

If $\mathcal{A}$ chooses $\left(v_{10}, v_{12}\right)$, then $\mathcal{B}$ chooses $\left(v_{9}, v_{12}\right)$.

In each case, the resulting graph satisfies:

1. $(z(G), p(G), t(G))=(n-12,1,2)$,

2. if $P(G)=\left\{u_{1}\right\}$ and $T(G)=\left\{u_{2}, u_{3}\right\}$, then, up to isomorphism, $\left(u_{2}, u_{3}\right) \in$ $E(G),\left(u_{1}, u_{2}\right) \in F(G)$, and

3. $\left(u_{1}, u_{3}\right) \in(E(G) \cup F(G))^{c}$.

Gameplay is identical in each case, so we may consider the first case. Call this graph $G_{(1)}^{5}$. 
- If $\mathcal{A}$ chooses $\left(v_{11}, v_{12}\right)$, then $\mathcal{B}$ chooses $\left(v_{10}, v_{11}\right)$. Call this graph $G_{(2)}^{5}$.

Case 4.2: $G_{n, 14}=G_{(2)}^{4} \cdot \mathcal{A}$ has five choices: $\left(v_{9}, v_{10}\right),\left(v_{9}, v_{12}\right),\left(v_{10}, v_{11}\right),\left(v_{10}, v_{12}\right)$, or $\left(v_{11}, v_{12}\right)$.

- If $\mathcal{A}$ chooses one of $\left(v_{9}, v_{10}\right)$ or $\left(v_{10}, v_{12}\right)$, then $\mathcal{B}$ chooses the other edge. Call this $\operatorname{graph} G_{(3)}^{5}$.

- If $\mathcal{A}$ chooses one of $\left(v_{9}, v_{12}\right)$ or $\left(v_{10}, v_{11}\right)$, then $\mathcal{B}$ chooses the other edge.

If $\mathcal{A}$ chooses $\left(v_{11}, v_{12}\right)$, then $\mathcal{B}$ chooses $\left(v_{9}, v_{12}\right)$.

In each case, the resulting graph satisfies:

1. $(z(G), p(G), t(G))=(n-12,1,2)$,

2. if $P(G)=\left\{u_{1}\right\}$ and $T(G)=\left\{u_{2}, u_{3}\right\}$, then $\left(u_{2}, u_{3}\right) \in E(G)$, and

3. all other pairs are in $(E(G) \cup F(G))^{c}$.

Gameplay is identical in each case, so we may consider the first case. Call this graph $G_{(4)}^{5}$.

Case 4.3: $G_{n, 14}=G_{(3)}^{4}$. $\mathcal{A}$ has four choices: $\left(v_{8}, v_{10}\right),\left(v_{8}, v_{12}\right),\left(v_{10}, v_{11}\right)$, or $\left(v_{10}, v_{12}\right)$.

- If $\mathcal{A}$ chooses one of $\left(v_{8}, v_{10}\right)$ or $\left(v_{10}, v_{12}\right)$, then $\mathcal{B}$ chooses the other edge. This graph satisfies:

1. $(z(G), p(G), t(G))=(n-12,2,0)$, and

2. if $P(G)=\left\{u_{1}, u_{2}\right\}$, then $\left(u_{1}, u_{2}\right) \in(E(G) \cup F(G))^{c}$.

Gameplay is identical to that of $G_{(3)}^{5}$.

- If $\mathcal{A}$ chooses one of $\left(v_{8}, v_{12}\right)$ or $\left(v_{10}, v_{11}\right)$, then $\mathcal{B}$ chooses the other edge. This graph satisfies:

1. $(z(G), p(G), t(G))=(n-12,1,2)$,

2. if $P(G)=\left\{u_{1}\right\}$ and $T(G)=\left\{u_{2}, u_{3}\right\}$, then $\left(u_{2}, u_{3}\right) \in E(G)$, and

3. all other pairs are in $(E(G) \cup F(G))^{c}$.

Gameplay is identical to that of $G_{(4)}^{5}$.

Case 4.4: $G_{n, 14}=G_{(4)}^{4} \cdot \mathcal{A}$ has five choices: $\left(v_{6}, v_{8}\right),\left(v_{6}, v_{11}\right),\left(v_{6}, v_{12}\right),\left(v_{8}, v_{12}\right)$, or $\left(v_{11}, v_{12}\right)$. 
- If $\mathcal{A}$ chooses $\left(v_{6}, v_{8}\right)$, then $\mathcal{B}$ chooses $\left(v_{7}, v_{12}\right)$.

If $\mathcal{A}$ chooses $\left(v_{6}, v_{12}\right)$, then $\mathcal{B}$ chooses $\left(v_{7}, v_{8}\right)$.

In each case, the resulting graph satisfies:

1. $(z(G), p(G), t(G))=(n-12,2,0)$, and

2. if $P(G)=\left\{u_{1}, u_{2}\right\}$, then $\left(u_{1}, u_{2}\right) \in(E(G) \cup F(G))^{c}$.

Gameplay is identical to that of $G_{(3)}^{5}$.

- If $\mathcal{A}$ chooses $\left(v_{6}, v_{11}\right)$, then $\mathcal{B}$ chooses $\left(v_{7}, v_{12}\right)$. Call this graph $G_{(5)}^{5}$.

- If $\mathcal{A}$ chooses one of $\left(v_{8}, v_{12}\right)$ or $\left(v_{11}, v_{12}\right)$, then $\mathcal{B}$ chooses the other edge. Call this $\operatorname{graph} G_{(6)}^{5}$.

Case 4.5: $G_{n, 14}=G_{(5)}^{4} \cdot \mathcal{A}$ has five choices: $\left(v_{8}, v_{9}\right),\left(v_{8}, v_{12}\right),\left(v_{9}, v_{11}\right),\left(v_{9}, v_{12}\right)$, or $\left(v_{11}, v_{12}\right)$.

- If $\mathcal{A}$ chooses $\left(v_{8}, v_{9}\right)$, then $\mathcal{B}$ chooses $\left(v_{10}, v_{12}\right)$. This graph satisfies:

1. $(z(G), p(G), t(G))=(n-12,2,0)$, and

2. if $P(G)=\left\{u_{1}, u_{2}\right\}$, then $\left(u_{1}, u_{2}\right) \in(E(G) \cup F(G))^{c}$.

Gameplay is identical to that of $G_{(3)}^{5}$.

- If $\mathcal{A}$ chooses one of $\left(v_{8}, v_{12}\right)$ or $\left(v_{9}, v_{11}\right)$, then $\mathcal{B}$ chooses the other edge. This graph satisfies:

1. $(z(G), p(G), t(G))=(n-12,1,2)$,

2. if $P(G)=\left\{u_{1}\right\}$ and $T(G)=\left\{u_{2}, u_{3}\right\}$, then $\left(u_{2}, u_{3}\right) \in F(G)$, and

3. all other pairs are in $(E(G) \cup F(G))^{c}$.

Gameplay is identical to that of $G_{(5)}^{5}$.

- If $\mathcal{A}$ chooses one of $\left(v_{9}, v_{12}\right)$ or $\left(v_{11}, v_{12}\right)$, then $\mathcal{B}$ chooses the other edge. Call this $\operatorname{graph} G_{(7)}^{5}$.

Case 4.6: $G_{n, 14}=G_{(6)}^{4}$. $\mathcal{A}$ has six choices: $\left(v_{8}, v_{10}\right),\left(v_{8}, v_{11}\right),\left(v_{8}, v_{12}\right),\left(v_{10}, v_{11}\right)$, $\left(v_{10}, v_{12}\right)$, or $\left(v_{11}, v_{12}\right)$.

- If $\mathcal{A}$ chooses $\left(v_{8}, v_{10}\right)$, then $\mathcal{B}$ chooses $\left(v_{9}, v_{12}\right)$.

If $\mathcal{A}$ chooses $\left(v_{8}, v_{12}\right)$, then $\mathcal{B}$ chooses $\left(v_{9}, v_{10}\right)$.

In each case, the resulting graph satisfies: 
1. $(z(G), p(G), t(G))=(n-12,2,0)$, and

2. if $P(G)=\left\{u_{1}, u_{2}\right\}$, then $\left(u_{1}, u_{2}\right) \in(E(G) \cup F(G))^{c}$.

Gameplay is identical to that of $G_{(3)}^{5}$.

- If $\mathcal{A}$ chooses one of $\left(v_{8}, v_{11}\right)$ or $\left(v_{10}, v_{12}\right)$, then $\mathcal{B}$ chooses the other edge. This graph satisfies:

1. $(z(G), p(G), t(G))=(n-12,1,2)$,

2. if $P(G)=\left\{u_{1}\right\}$ and $T(G)=\left\{u_{2}, u_{3}\right\}$, then $\left(u_{2}, u_{3}\right) \in F(G)$, and

3. all other pairs are in $(E(G) \cup F(G))^{c}$.

Gameplay is identical to that of $G_{(5)}^{5}$.

- If $\mathcal{A}$ chooses one of $\left(v_{10}, v_{11}\right)$ or $\left(v_{11}, v_{12}\right)$, then $\mathcal{B}$ chooses the other edge. This graph satisfies:

1. $(z(G), p(G), t(G))=(n-12,1,2)$,

2. if $P(G)=\left\{u_{1}\right\}$ and $T(G)=\left\{u_{2}, u_{3}\right\}$, then $\left(u_{2}, u_{3}\right) \in E(G)$, and

3. all other pairs are in $(E(G) \cup F(G))^{c}$.

Gameplay is identical to that of $G_{(4)}^{5}$.

Case 4.7: $G_{n, 14}=G_{(7)}^{4} \cdot \mathcal{A}$ must choose $\left(v_{7}, v_{11}\right)$. Then $\mathcal{B}$ chooses $\left(v_{10}, v_{12}\right)$. This graph satisfies:

1. $(z(G), p(G), t(G))=(n-12,2,0)$, and

2. if $P(G)=\left\{u_{1}, u_{2}\right\}$, then $\left(u_{1}, u_{2}\right) \in(E(G) \cup F(G))^{c}$.

Gameplay is identical to that of $G_{(3)}^{5}$.

Case 4.8: $G_{n, 14}=G_{(8)}^{4}$. $\mathcal{A}$ has seven choices: $\left(v_{4}, v_{9}\right),\left(v_{4}, v_{11}\right),\left(v_{4}, v_{12}\right),\left(v_{9}, v_{10}\right)$, $\left(v_{9}, v_{12}\right),\left(v_{10}, v_{12}\right)$, or $\left(v_{11}, v_{12}\right)$.

- If $\mathcal{A}$ chooses $\left(v_{4}, v_{9}\right)$, then $\mathcal{B}$ chooses $\left(v_{7}, v_{12}\right)$.

If $\mathcal{A}$ chooses $\left(v_{4}, v_{12}\right)$, then $\mathcal{B}$ chooses $\left(v_{7}, v_{9}\right)$.

In each case, the resulting graph satisfies:

1. $(z(G), p(G), t(G))=(n-12,1,2)$,

2. if $P(G)=\left\{u_{1}\right\}$ and $T(G)=\left\{u_{2}, u_{3}\right\}$, then $\left(u_{2}, u_{3}\right) \in E(G)$, and

3. all other pairs are in $(E(G) \cup F(G))^{c}$.

Gameplay is identical to that of $G_{(4)}^{5}$. 
- If $\mathcal{A}$ chooses one of $\left(v_{4}, v_{11}\right)$ or $\left(v_{9}, v_{12}\right)$, then $\mathcal{B}$ chooses the other edge. If $\mathcal{A}$ chooses one of $\left(v_{9}, v_{10}\right)$ or $\left(v_{11}, v_{12}\right)$, then $\mathcal{B}$ chooses the other edge. In each case, the resulting graph satisfies:

1. $(z(G), p(G), t(G))=(n-12,1,2)$,

2. if $P(G)=\left\{u_{1}\right\}$ and $T(G)=\left\{u_{2}, u_{3}\right\}$, then $\left(u_{2}, u_{3}\right) \in F(G)$, and

3. all other pairs are in $(E(G) \cup F(G))^{c}$.

Gameplay is identical to that of $G_{(5)}^{5}$.

- If $\mathcal{A}$ chooses $\left(v_{10}, v_{12}\right)$, then $\mathcal{B}$ chooses $\left(v_{9}, v_{12}\right)$. Call this graph $G_{(8)}^{5}$.

Finally, we look at the graphs $G_{(i)}^{5}$ for $1 \leq i \leq 8$.

Case 5.1: $G_{n, 16}=G_{(1)}^{5}$. $\mathcal{A}$ has four choices: $\left(v_{10}, v_{12}\right),\left(v_{10}, v_{13}\right),\left(v_{11}, v_{13}\right)$, or $\left(v_{12}, v_{13}\right)$.

- If $\mathcal{A}$ chooses one of $\left(v_{10}, v_{12}\right)$ or $\left(v_{11}, v_{13}\right)$, then $\mathcal{B}$ chooses the other edge. The resulting graph is in $\mathcal{L}_{1}(n-13)(b)$.

- If $\mathcal{A}$ chooses one of $\left(v_{10}, v_{13}\right)$ or $\left(v_{12}, v_{13}\right)$, then $\mathcal{B}$ chooses the other edge. The resulting graph is in $\mathcal{L}_{1}(n-13)(c)$.

Case 5.2: $G_{n, 16}=G_{(2)}^{5}$. $\mathcal{A}$ has two choices: $\left(v_{9}, v_{13}\right)$ or $\left(v_{12}, v_{13}\right)$. $\mathcal{B}$ chooses whichever edge $\mathcal{A}$ did not. The resulting graph is in $\mathcal{L}_{1}(n-13)(d)$.

Case 5.3: $G_{n, 16}=G_{(3)}^{5}$. $\mathcal{A}$ has two choices: $\left(v_{11}, v_{12}\right)$ or $\left(v_{11}, v_{13}\right)$. $\mathcal{B}$ chooses whichever edge $\mathcal{A}$ did not. The resulting graph is in $\mathcal{L}_{1}(n-13)(a)$.

Case 5.4: $G_{n, 16}=G_{(4)}^{5}$. $\mathcal{A}$ has three choices: $\left(v_{10}, v_{12}\right),\left(v_{10}, v_{13}\right)$, or $\left(v_{12}, v_{13}\right)$.

- If $\mathcal{A}$ chooses $\left(v_{10}, v_{12}\right)$, then $\mathcal{B}$ chooses $\left(v_{11}, v_{13}\right)$. The resulting graph is in $\mathcal{L}_{1}(n-$ $13)(b)$.

- If $\mathcal{A}$ chooses one of $\left(v_{10}, v_{13}\right)$ or $\left(v_{12}, v_{13}\right)$, then $\mathcal{B}$ chooses the other edge. The resulting graph is in $\mathcal{L}_{1}(n-13)(d)$.

Case 5.5: $G_{n, 16}=G_{(5)}^{5}$. $\mathcal{A}$ has three choices: $\left(v_{8}, v_{12}\right),\left(v_{8}, v_{13}\right)$, or $\left(v_{12}, v_{13}\right)$.

If $\mathcal{A}$ chooses $\left(v_{8}, v_{12}\right)$, then $\mathcal{B}$ chooses $\left(v_{11}, v_{13}\right)$.

If $\mathcal{A}$ chooses $\left(v_{8}, v_{13}\right)$, then $\mathcal{B}$ chooses $\left(v_{11}, v_{12}\right)$.

If $\mathcal{A}$ chooses $\left(v_{12}, v_{13}\right)$, then $\mathcal{B}$ chooses $\left(v_{8}, v_{12}\right)$.

In each case, the resulting graph is in $\mathcal{L}_{1}(n-13)(b)$.

Case 5.6: $G_{n, 16}=G_{(6)}^{5}$. $\mathcal{A}$ has three choices: $\left(v_{6}, v_{11}\right),\left(v_{6}, v_{13}\right)$, or $\left(v_{11}, v_{13}\right)$.

If $\mathcal{A}$ chooses $\left(v_{6}, v_{11}\right)$, then $\mathcal{B}$ chooses $\left(v_{7}, v_{13}\right)$.

If $\mathcal{A}$ chooses $\left(v_{6}, v_{13}\right)$, then $\mathcal{B}$ chooses $\left(v_{7}, v_{11}\right)$.

If $\mathcal{A}$ chooses $\left(v_{11}, v_{13}\right)$, then $\mathcal{B}$ chooses $\left(v_{6}, v_{12}\right)$.

In each case, the resulting graph is in $\mathcal{L}_{1}(n-13)(b)$.

Case 5.7: $G_{n, 16}=G_{(7)}^{5}$. $\mathcal{A}$ has four choices: $\left(v_{8}, v_{10}\right),\left(v_{8}, v_{12}\right),\left(v_{8}, v_{13}\right)$, or $\left(v_{11}, v_{13}\right)$. 
- If $\mathcal{A}$ chooses one of $\left(v_{8}, v_{10}\right)$ or $\left(v_{11}, v_{13}\right)$, then $\mathcal{B}$ chooses the other edge. The resulting graph is in $\mathcal{L}_{1}(n-13)(a)$.

- If $\mathcal{A}$ chooses $\left(v_{8}, v_{12}\right)$, then $\mathcal{B}$ chooses $\left(v_{10}, v_{13}\right)$.

If $\mathcal{A}$ chooses $\left(v_{8}, v_{13}\right)$, then $\mathcal{B}$ chooses $\left(v_{10}, v_{11}\right)$.

In each case, the resulting graph is in $\mathcal{L}_{1}(n-13)(b)$.

Case 5.8: $G_{n, 16}=G_{(8)}^{5}$. $\mathcal{A}$ has two choices: $\left(v_{4}, v_{11}\right)$ or $\left(v_{4}, v_{13}\right)$.

If $\mathcal{A}$ chooses $\left(v_{4}, v_{11}\right)$, then $\mathcal{B}$ chooses $\left(v_{7}, v_{13}\right)$.

If $\mathcal{A}$ chooses $\left(v_{4}, v_{13}\right)$, then $\mathcal{B}$ chooses $\left(v_{7}, v_{11}\right)$.

In each case, the resulting graph is in $\mathcal{L}_{1}(n-13)(b)$.

Lemma 5.3. For $n \geq 3$, in $\Gamma_{\{B 3, T, C\}}(n)$, if either player chooses an edge $e_{k}$ that creates a graph $G_{n, k} \in \mathcal{L}_{1}(z)$, then

- when $z \geq 1$, that player can choose an edge $e_{k+2}$ so that $G_{n, k+2} \in\left(\mathcal{L}_{2}(z-1) \cup \mathcal{L}_{3}(z-\right.$ 1)), or

- when $z \geq 4$, that player can choose edges $e_{k+2}, e_{k+4}, e_{k+6}$ so that $G_{n, k+6} \in\left(\mathcal{L}_{2}(z-\right.$ 4) $\left.\cup \mathcal{L}_{3}(z-4)\right)$.

Proof. Let $n \geq 3$. Suppose the edges $e_{1}, \ldots, e_{k-1}$ have already been chosen. Suppose player $\mathcal{A}$ chooses the $k$ th edge so that $G_{n, k} \in \mathcal{L}_{1}(z)$ with $z \geq 1$. Let $w_{1} \in Z\left(G_{n, k}\right)$. Each vertex $v \notin\left(Z\left(G_{n, k}\right) \cup P\left(G_{n, k}\right) \cup T\left(G_{n, k}\right)\right)$ has $\operatorname{deg}_{G_{n, k}}(v)=3$ and is out of play. The strategy described here will work for $\mathcal{B}$ as well.

$\boldsymbol{G}_{\boldsymbol{n}, \boldsymbol{k}} \in \mathcal{L}_{\mathbf{1}}(\boldsymbol{z})(\boldsymbol{a})$ :

Let $P\left(G_{n, k}\right)=\left\{u_{1}\right\}$ and $T\left(G_{n, k}\right)=\left\{u_{2}\right\}$ with $\left(u_{1}, u_{2}\right) \in F\left(G_{n, k}\right)$. Up to isomorphism, $\mathcal{B}$ has two choices: $\left(u_{1}, w_{1}\right)$ or $\left(u_{2}, w_{1}\right)$. $\mathcal{A}$ chooses whichever edge $\mathcal{B}$ did not. Now $u_{2}$ is out of play, $z\left(G_{n, k+2}\right)=z\left(G_{n, k}\right)-1=z-1, p\left(G_{n, k+2}\right)=0, T\left(G_{n, k+2}\right)=\left\{u_{1}, w_{1}\right\}$, and $\left(u_{1}, w_{1}\right) \in E_{k+2}$. So $G_{n, k+2} \in \mathcal{L}_{2}(z-1)(b)$.

$\boldsymbol{G}_{n, k} \in \mathcal{L}_{1}(z)(b):$

Let $P\left(G_{n, k}\right)=\left\{u_{1}\right\}$ and $T\left(G_{n, k}\right)=\left\{u_{2}\right\}$ with $\left(u_{1}, u_{2}\right) \notin\left(E_{k} \cup F\left(G_{n, k}\right)\right)$. Up to isomorphism, $\mathcal{B}$ has three choices: $\left(u_{1}, u_{2}\right),\left(u_{1}, w_{1}\right)$, or $\left(u_{2}, w_{1}\right)$.

- If $\mathcal{B}$ chooses $\left(u_{1}, u_{2}\right)$ then $\mathcal{A}$ chooses $\left(u_{1}, w_{1}\right)$. Now $u_{1}, u_{2}$ are out of play, $z\left(G_{n, k+2}\right)=$ $z\left(G_{n, k}\right)-1=z-1, P\left(G_{n, k+2}\right)=\left\{w_{1}\right\}$, and $t\left(G_{n, k+2}\right)=0$. So $G_{n, k+2} \in \mathcal{L}_{3}(z-1)(a)$.

- If $\mathcal{B}$ chooses one of $\left(u_{1}, w_{1}\right)$ or $\left(u_{2}, w_{1}\right)$, then $\mathcal{A}$ chooses the other edge. This case is identical to the above case $G_{n, k} \in \mathcal{L}_{1}(z)(a)$ and $G_{n, k+2} \in \mathcal{L}_{2}(z-1)(b)$.

$G_{n, k} \in \mathcal{L}_{1}(z)(c):$

Let $T\left(G_{n, k}\right)=\left\{u_{1}, u_{2}, u_{3}\right\}$ with $\left(u_{1}, u_{2}\right) \in E_{k}$ and $\left(u_{1}, u_{3}\right),\left(u_{2}, u_{3}\right) \in F\left(G_{n, k}\right)$. Note that $p\left(G_{n, k}\right)=0$. Up to isomorphism, $\mathcal{B}$ has two choices: $\left(u_{1}, w_{1}\right)$ or $\left(u_{3}, w_{1}\right)$. $\mathcal{A}$ chooses whichever edge $\mathcal{B}$ did not. Now $u_{1}, u_{3}$ are out of play, $z\left(G_{n, k+2}\right)=z\left(G_{n, k}\right)-1=z-1$, $p\left(G_{n, k+2}\right)=0, T\left(G_{n, k+2}\right)=\left\{u_{2}, w_{1}\right\}$, and $\left(u_{2}, w_{1}\right) \in F\left(G_{n, k+2}\right)$. So $G_{n, k+2} \in \mathcal{L}_{2}(z-1)(c)$. 
$G_{n, k} \in \mathcal{L}_{1}(z)(d)$

Let $u_{1}, u_{2}, u_{3} \in T(G)$, with $\left(u_{1}, u_{2}\right) \in E,\left(u_{1}, u_{3}\right) \in F(G)$, and $\left(u_{2}, u_{3}\right) \notin(E \cup F(G))$. Note that $p\left(G_{n, k}\right)=0$. Up to isomorphism, $\mathcal{B}$ has four choices: $\left(u_{1}, w_{1}\right),\left(u_{2}, u_{3}\right),\left(u_{2}, w_{1}\right)$, or $\left(u_{3}, w_{1}\right)$.

- If $\mathcal{B}$ chooses one of $\left(u_{1}, w_{1}\right)$ or $\left(u_{2}, u_{3}\right)$, then $\mathcal{A}$ chooses the other edge. Now $u_{1}, u_{2}, u_{3}$ are out of play, $z\left(G_{n, k+2}\right)=z\left(G_{n, k}\right)-1=z-1, P\left(G_{n, k+2}\right)=\left\{w_{1}\right\}$, and $t\left(G_{n, k+2}\right)=$ 0 . So $G_{n, k+2} \in \mathcal{L}_{3}(z-1)(a)$.

- If $\mathcal{B}$ chooses $\left(u_{2}, w_{1}\right)$, then $\mathcal{A}$ chooses $\left(u_{3}, w_{1}\right)$. Now $u_{2}, u_{3}$ are out of play, $z\left(G_{n, k+2}\right)=$ $z\left(G_{n, k}\right)-1=z-1, p\left(G_{n, k+2}\right)=0, T\left(G_{n, k+2}\right)=\left\{u_{1}, w_{1}\right\}$, and $\left(u_{1}, w_{1}\right) \in F\left(G_{n, k+2}\right)$. So $G_{n, k+2} \in \mathcal{L}_{2}(z-1)(c)$.

- If $\mathcal{B}$ chooses $\left(u_{3}, w_{1}\right)$, then $\mathcal{A}$ chooses $\left(u_{1}, w_{1}\right)$. Now $u_{1}, u_{3}$ are out of play, $z\left(G_{n, k+2}\right)=$ $z\left(G_{n, k}\right)-1=z-1, p\left(G_{n, k+2}\right)=0, T\left(G_{n, k+2}\right)=\left\{u_{2}, w_{1}\right\}$, and $\left(u_{2}, w_{1}\right) \in F\left(G_{n, k+2}\right)$. So $G_{n, k+2} \in \mathcal{L}_{2}(z-1)(c)$.

Now suppose player $\mathcal{A}$ chooses the $k$ th edge so that $G_{n, k} \in \mathcal{L}_{1}(z)$ with $z \geq 4$. Let $w_{1}, w_{2}, w_{3}, w_{4} \in Z\left(G_{n, k}\right)$.

$G_{n, k} \in \mathcal{L}_{1}(z)(a)$ :

$\mathcal{A}$ chooses the edge $e_{k+2}$ as above. Now $G_{n, k+2} \in \mathcal{L}_{2}(z-1)(b)$ with $z\left(G_{n, k+2}\right)=z-1$, $p\left(G_{n, k+2}\right)=0, T\left(G_{n, k+2}\right)=\left\{u_{1}, w_{1}\right\}$, and $\left(u_{1}, w_{1}\right) \in E_{k+2}$. Up to game play equivalence, $\mathcal{B}$ must choose $\left(u_{1}, w_{2}\right)$. Then $\mathcal{A}$ chooses $\left(w_{1}, w_{3}\right)$. Now $\mathcal{B}$ has two choices: $\left(w_{2}, w_{3}\right)$ or $\left(w_{2}, w_{4}\right)$. $\mathcal{A}$ chooses whichever edge $\mathcal{B}$ did not. Now $u_{1}, w_{1}, w_{2}$ are out of play, $z\left(G_{n, k+6}\right)=$ $z\left(G_{n, k}\right)-4=z-4, P\left(G_{n, k+6}\right)=\left\{w_{4}\right\}, T\left(G_{n, k+6}\right)=\left\{w_{3}\right\}$, and $\left(w_{3}, w_{4}\right) \in F\left(G_{n, k+6}\right)$. So $G_{n, k+6} \in \mathcal{L}_{2}(z-4)(a)$.

$G_{n, k} \in \mathcal{L}_{1}(z)(b):$

$\mathcal{A}$ chooses the edge $e_{k+2}$ as above. Now we have two cases.

1. $G_{n, k+2} \in \mathcal{L}_{3}(z-1)(a)$ with $z\left(G_{n, k+2}\right)=z-1, P\left(G_{n, k+2}\right)=\left\{w_{1}\right\}$, and $t\left(G_{n, k+2}\right)=0$. Up to isomorphism, $\mathcal{B}$ must choose $\left(w_{1}, w_{2}\right)$. Then $\mathcal{A}$ chooses $\left(w_{1}, w_{3}\right)$. $\mathcal{B}$ must choose $\left(w_{2}, w_{4}\right)$. $\mathcal{A}$ chooses $\left(w_{3}, w_{4}\right)$. Now $w_{1}$ is out of play, $z\left(G_{n, k+6}\right)=z\left(G_{n, k}\right)-4=$ $z-4, p\left(G_{n, k+6}\right)=0, T\left(G_{n, k+6}\right)=\left\{w_{2}, w_{3}, w_{4}\right\}$, and $\left(w_{2}, w_{4}\right),\left(w_{3}, w_{4}\right) \in E_{k+6}$. So $G_{n, k+6} \in \mathcal{L}_{3}(z-4)(b)$.

2. $G_{n, k+2} \in \mathcal{L}_{2}(z-1)(b)$ with $z\left(G_{n, k+2}\right)=z-1, p\left(G_{n, k+2}\right)=0, T\left(G_{n, k+2}\right)=\left\{u_{1}, w_{1}\right\}$, and $\left(u_{1}, w_{1}\right) \in E_{k+2}$. This case is identical to $G_{n, k+2}$ in $\mathcal{L}_{1}(z)(a)$ above and $\mathcal{A}$ follows the same pattern.

$G_{n, k} \in \mathcal{L}_{1}(z)(c):$

$\mathcal{A}$ chooses the edge $e_{k+2}$ as above. Now $G_{n, k+2} \in \mathcal{L}_{2}(z-1)(c)$ with $z\left(G_{n, k+2}\right)=$ $z-1, p\left(G_{n, k+2}\right)=0, T\left(G_{n, k+2}\right)=\left\{u_{2}, w_{1}\right\}$, and $\left(u_{2}, w_{1}\right) \in F\left(G_{n, k+2}\right)$. Up to game play equivalence, $\mathcal{B}$ must choose $\left(u_{2}, w_{2}\right)$. Then $\mathcal{A}$ chooses $\left(w_{1}, w_{3}\right)$. Now $\mathcal{B}$ has two choices: $\left(w_{2}, w_{3}\right)$ or $\left(w_{2}, w_{4}\right)$. $\mathcal{A}$ chooses whichever edge $\mathcal{B}$ did not. Now $u_{1}, w_{1}, w_{2}$ are 
out of play, $z\left(G_{n, k+6}\right)=z\left(G_{n, k}\right)-4=z-4, P\left(G_{n, k+6}\right)=\left\{w_{4}\right\}, T\left(G_{n, k+6}\right)=\left\{w_{3}\right\}$, and $\left(w_{3}, w_{4}\right) \in F\left(G_{n, k+6}\right)$. So $G_{n, k+6} \in \mathcal{L}_{2}(z-4)(a)$.

$G_{n, k} \in \mathcal{L}_{1}(z)(d)$ :

$\mathcal{A}$ chooses the edge $e_{k+2}$ as above. Now we have three cases.

1. $G_{n, k+2} \in \mathcal{L}_{3}(z-1)(a)$ with $z\left(G_{n, k+2}\right)=z-1, P\left(G_{n, k+2}\right)=\left\{w_{1}\right\}$, and $t\left(G_{n, k+2}\right)=0$. This case is identical to $G_{n, k+2}$ in the first case of $\mathcal{L}_{1}(z)(b)$ above and $\mathcal{A}$ follows the same pattern.

2. $G_{n, k+2} \in \mathcal{L}_{2}(z-1)(c)$ with $z\left(G_{n, k+2}\right)=z-1, p\left(G_{n, k+2}\right)=0, T\left(G_{n, k+2}\right)=\left\{u_{1}, w_{1}\right\}$, and $\left(u_{1}, w_{1}\right) \in F\left(G_{n, k+2}\right)$. This case is identical to $G_{n, k+2}$ in $\mathcal{L}_{1}(z)(c)$ above and $\mathcal{A}$ follows the same pattern.

3. $G_{n, k+2} \in \mathcal{L}_{2}(z-1)(c)$ with $z\left(G_{n, k+2}\right)=z-1, p\left(G_{n, k+2}\right)=0, T\left(G_{n, k+2}\right)=\left\{u_{2}, w_{1}\right\}$, and $\left(u_{2}, w_{1}\right) \in F\left(G_{n, k+2}\right)$. This case is identical to $G_{n, k+2}$ in $\mathcal{L}_{1}(z)(c)$ above and $\mathcal{A}$ follows the same pattern.

Lemma 5.4. For $n \geq 3$, in $\Gamma_{\{B 3, T, C\}}(n)$, if either player chooses an edge $e_{k}$ that creates a graph $G_{n, k} \in \mathcal{L}_{2}(z)$ for some $z \equiv 0(\bmod 4)$, then that player has a winning strategy.

Proof. Let $n \geq 3$. Suppose the edges $e_{1}, \ldots, e_{k-1}$ have already been chosen. Suppose player $\mathcal{A}$ chooses the $k$ th edge so that $G_{n, k} \in \mathcal{L}_{2}(z)$ with $z \equiv 0(\bmod 4)$. The strategy described here will work for $\mathcal{B}$ as well. We proceed by induction.

Base case: $z=0$. In each graph in $\mathcal{L}_{2}(0)$, no new edge can be chosen. Since $\mathcal{A}$ chose the last edge, $\mathcal{A}$ wins.

Assume for induction that if $\mathcal{A}$ chooses an edge $e_{k}$ that creates a graph $G_{n, k} \in \mathcal{L}_{2}(4 m)$ for some $m \geq 0$, then $\mathcal{A}$ has a winning strategy. Suppose $z=4(m+1)$. Let $w_{1}, w_{2}, w_{3}, w_{4} \in$ $Z\left(G_{n, k}\right)$.

$G_{n, k} \in \mathcal{L}_{2}(z)(a)$

Let $P\left(G_{n, k}\right)=\left\{u_{1}\right\}$ and $T\left(G_{n, k}\right)=\left\{u_{2}\right\}$ with $\left(u_{1}, u_{2}\right) \in F\left(G_{n, k}\right)$. Up to isomorphism, $\mathcal{B}$ has two choices: $\left(u_{1}, w_{1}\right)$ or $\left(u_{2}, w_{1}\right)$. $\mathcal{A}$ chooses whichever edge $\mathcal{B}$ did not. Up to game play equivalence, $\mathcal{B}$ must choose $\left(u_{1}, w_{2}\right)$. Then $\mathcal{A}$ chooses $\left(w_{1}, w_{3}\right)$. Now $\mathcal{B}$ has two choices: $\left(w_{2}, w_{3}\right)$ or $\left(w_{2}, w_{4}\right)$. $\mathcal{A}$ chooses whichever edge $\mathcal{B}$ did not. Now $u_{1}, u_{2}, w_{1}, w_{2}$ are out of play, $z\left(G_{n, k+6}\right)=z\left(G_{n, k}\right)-4=4 m, P\left(G_{n, k+6}\right)=\left\{w_{4}\right\}, T\left(G_{n, k+6}\right)=\left\{w_{3}\right\}$, and $\left(w_{3}, w_{4}\right) \in F\left(G_{n, k+6}\right)$. Thus $G_{n, k+6} \in \mathcal{L}_{2}(4 m)(a)$ and $\mathcal{A}$ wins by induction.

$G_{n, k} \in \mathcal{L}_{2}(z)(b)$ :

Let $T\left(G_{n, k}\right)=\left\{u_{1}, u_{2}\right\}$ with $\left(u_{1}, u_{2}\right) \in E_{k}$. Note that $p\left(G_{n, k}\right)=0$. Up to game play equivalence, $\mathcal{B}$ must choose $\left(u_{1}, w_{1}\right)$. $\mathcal{A}$ chooses $\left(u_{2}, w_{2}\right)$. $\mathcal{B}$ has two choices: $\left(w_{1}, w_{2}\right)$ or $\left(w_{1}, w_{3}\right) . \mathcal{A}$ chooses whichever edge $\mathcal{B}$ did not. $\mathcal{B}$ again has two choices: $\left(w_{2}, w_{4}\right)$ or $\left(w_{3}, w_{4}\right)$. $\mathcal{A}$ chooses whichever edge $\mathcal{B}$ did not. Now $u_{1}, u_{2}, w_{1}, w_{2}$ are out of play, $z\left(G_{n, k+6}\right)=z\left(G_{n, k}\right)-4=4 m, p\left(G_{n, k+6}\right)=0, T\left(G_{n, k+6}\right)=\left\{w_{3}, w_{4}\right\}$, and $\left(w_{3}, w_{4}\right) \in E_{k+6}$. Thus $G_{n, k+6} \in \mathcal{L}_{2}(4 m)(b)$ and $\mathcal{A}$ wins by induction.

$G_{n, k} \in \mathcal{L}_{2}(z)(c)$ : 
Let $T\left(G_{n, k}\right)=\left\{u_{1}, u_{2}\right\}$ with $\left(u_{1}, u_{2}\right) \in F\left(G_{n, k}\right)$. Note that $p\left(G_{n, k}\right)=0$. Up to game play equivalence, $\mathcal{B}$ must choose $\left(u_{1}, w_{1}\right)$. $\mathcal{A}$ chooses $\left(u_{2}, w_{2}\right)$. Now this case is identical to the above case $G_{n, k} \in \mathcal{L}_{2}(z)(b)$ and $\mathcal{A}$ wins.

Lemma 5.5. For $n \geq 3$, in $\Gamma_{\{B 3, T, C\}}(n)$, if either player chooses an edge $e_{k}$ that creates a graph $G_{n, k} \in \mathcal{L}_{3}(z)$ for some $z \equiv 0(\bmod 8)$, then that player has a winning strategy.

Proof. Let $n \geq 3$. Suppose the edges $e_{1}, \ldots, e_{k-1}$ have already been chosen. Suppose player $\mathcal{A}$ chooses the $k$ th edge so that $G_{n, k} \in \mathcal{L}_{3}(z)$ with $z \equiv 0(\bmod 8)$. The strategy described here will work for $\mathcal{B}$ as well. We proceed by induction.

Base case: $z=0$. In each graph in $\mathcal{L}_{3}(0)$, no new edge can be chosen. Since $\mathcal{A}$ chose the last edge, $\mathcal{A}$ wins.

Assume for induction that if $\mathcal{A}$ chooses an edge $e_{k}$ that creates a graph $G_{n, k} \in \mathcal{L}_{3}(8 m)$ for some $m \geq 0$, then $\mathcal{A}$ has a winning strategy. Suppose $z=8(m+1)$. Let $w_{1}, w_{2}, \ldots, w_{8} \in Z\left(G_{n, k}\right)$.

$G_{n, k} \in \mathcal{L}_{3}(z)(a)$ :

Let $P\left(G_{n, k}\right)=\left\{u_{1}\right\}$. Note that $t\left(G_{n, k}\right)=0$. Up to isomorphism, $\mathcal{B}$ must choose $\left(u_{1}, w_{1}\right)$. $\mathcal{A}$ chooses $\left(u_{1}, w_{2}\right)$. $\mathcal{B}$ must choose $\left(w_{1}, w_{3}\right)$. $\mathcal{A}$ chooses $\left(w_{2}, w_{3}\right) . \mathcal{B}$ has two choices: $\left(w_{1}, w_{4}\right)$ or $\left(w_{3}, w_{4}\right)$.

- If $\mathcal{B}$ chooses $\left(w_{1}, w_{4}\right)$ then $\mathcal{A}$ chooses $\left(w_{3}, w_{5}\right)$.

- If $\mathcal{B}$ chooses $\left(w_{3}, w_{4}\right)$ then $\mathcal{A}$ chooses $\left(w_{1}, w_{5}\right)$.

The two resulting graphs are isomorphic, so consider the first case. Now $\mathcal{B}$ has five choices: $\left(w_{2}, w_{4}\right),\left(w_{2}, w_{6}\right),\left(w_{4}, w_{5}\right),\left(w_{4}, w_{6}\right)$, or $\left(w_{5}, w_{6}\right)$.

- If $\mathcal{B}$ chooses one of $\left(w_{2}, w_{4}\right)$ or $\left(w_{4}, w_{6}\right)$, then $\mathcal{A}$ chooses the other edge.

$\mathcal{B}$ has two choices: $\left(w_{5}, w_{6}\right)$ or $\left(w_{5}, w_{7}\right)$. $\mathcal{A}$ chooses whichever edge $\mathcal{B}$ did not. $\mathcal{B}$ again has two choices: $\left(w_{6}, w_{8}\right)$ or $\left(w_{7}, w_{8}\right)$. $\mathcal{A}$ chooses whichever edge $\mathcal{B}$ did not. Now $u_{1}, w_{1}, w_{2}, \ldots, w_{6}$ are out of play, $z\left(G_{n, k+12}\right)=z\left(G_{n, k}\right)-8=8 m, p\left(G_{n, k+12}\right)=0$, $T\left(G_{n, k+12}\right)=\left\{w_{7}, w_{8}\right\}$, and $\left(w_{7}, w_{8}\right) \in E_{k+12}$. Thus $G_{n, k+12} \in \mathcal{L}_{2}(8 m)(b)$ and $\mathcal{A}$ wins by Lemma 5.4 .

- If $\mathcal{B}$ chooses one of $\left(w_{2}, w_{6}\right)$ or $\left(w_{4}, w_{5}\right)$, then $\mathcal{A}$ chooses the other edge.

If $\mathcal{B}$ chooses $\left(w_{5}, w_{6}\right)$, then $\mathcal{A}$ chooses $\left(w_{2}, w_{6}\right)$.

The two resulting graphs are isomorphic, so consider the first case. $\mathcal{B}$ has three choices: $\left(w_{4}, w_{6}\right),\left(w_{4}, w_{7}\right)$, or $\left(w_{6}, w_{7}\right)$.

- If $\mathcal{B}$ chooses $\left(w_{4}, w_{6}\right)$ then $\mathcal{A}$ chooses $\left(w_{5}, w_{7}\right)$.

- If $\mathcal{B}$ chooses $\left(w_{4}, w_{7}\right)$ then $\mathcal{A}$ chooses $\left(w_{5}, w_{6}\right)$.

- If $\mathcal{B}$ chooses $\left(w_{6}, w_{7}\right)$ then $\mathcal{A}$ chooses $\left(w_{4}, w_{6}\right)$. 
In each case, the resulting graph is in $\mathcal{L}_{1}(8 m+1)(b)$ and gameplay is identical, so without loss of generality we may consider the first case. $\mathcal{B}$ again has three choices: $\left(w_{6}, w_{7}\right),\left(w_{6}, w_{8}\right)$, or $\left(w_{7}, w_{8}\right)$.

- If $\mathcal{B}$ chooses $\left(w_{6}, w_{7}\right)$ then $\mathcal{A}$ chooses $\left(w_{7}, w_{8}\right)$. Now $u_{1}, w_{1}, w_{2}, \ldots, w_{7}$ are out of play, $z\left(G_{n, k+12}\right)=z\left(G_{n, k}\right)-8=8 m, P\left(G_{n, k+12}\right)=\left\{w_{8}\right\}$, and $t\left(G_{n, k+12}\right)=0$. Thus $G_{n, k+12} \in \mathcal{L}_{3}(8 m)(a)$ and $\mathcal{A}$ wins by induction.

- If $\mathcal{B}$ chooses one of $\left(w_{6}, w_{8}\right)$ or $\left(w_{7}, w_{8}\right)$, then $\mathcal{A}$ chooses the other edge. Now $u_{1}, w_{1}, w_{2}, \ldots, w_{6}$ are out of play, $z\left(G_{n, k+12}\right)=z\left(G_{n, k}\right)-8=8 m, p\left(G_{n, k+12}\right)=$ $0, T\left(G_{n, k+12}\right)=\left\{w_{7}, w_{8}\right\}$, and $\left(w_{7}, w_{8}\right) \in E_{k+12}$. Thus $G_{n, k+12} \in \mathcal{L}_{2}(8 m)(b)$ and $\mathcal{A}$ wins by Lemma 5.4 .

$G_{n, k} \in \mathcal{L}_{3}(z)(b):$

Let $T\left(G_{n, k}\right)=\left\{u_{1}, u_{2}, u_{3}\right\}$ with $\left(u_{1}, u_{2}\right),\left(u_{1}, u_{3}\right) \in E_{k}$. Note that $p\left(G_{n, k}\right)=0$ and $\left(u_{2}, u_{3}\right) \in F\left(G_{n, k}\right)$. Up to game play equivalence, $\mathcal{B}$ has two choices: $\left(u_{1}, w_{1}\right)$ or $\left(u_{2}, w_{1}\right)$.

- If $\mathcal{B}$ chooses $\left(u_{1}, w_{1}\right)$ then $\mathcal{A}$ chooses $\left(u_{2}, w_{2}\right)$.

- If $\mathcal{B}$ chooses $\left(u_{2}, w_{1}\right)$ then $\mathcal{A}$ chooses $\left(u_{1}, w_{2}\right)$.

The two resulting graphs are isomorphic, so consider the first case. Now $\mathcal{B}$ has five choices: $\left(u_{3}, w_{2}\right),\left(u_{3}, w_{3}\right),\left(w_{1}, w_{2}\right),\left(w_{1}, w_{3}\right)$, or $\left(w_{2}, w_{3}\right)$.

- If $\mathcal{B}$ chooses one of $\left(u_{3}, w_{2}\right)$ or $\left(w_{2}, w_{3}\right)$, then $\mathcal{A}$ chooses the other edge. $\mathcal{B}$ has two choices: $\left(w_{1}, w_{3}\right)$ or $\left(w_{1}, w_{4}\right)$. $\mathcal{A}$ chooses whichever edge $\mathcal{B}$ did not. $\mathcal{B}$ again has two choices: $\left(w_{3}, w_{5}\right)$ or $\left(w_{4}, w_{5}\right)$. $\mathcal{A}$ chooses whichever edge $\mathcal{B}$ did not. $\mathcal{B}$ must choose $\left(w_{4}, w_{6}\right)$. $\mathcal{A}$ chooses $\left(w_{5}, w_{7}\right)$. $\mathcal{B}$ has two choices: $\left(w_{6}, w_{7}\right)$ or $\left(w_{6}, w_{8}\right)$. $\mathcal{A}$ chooses whichever edge $\mathcal{B}$ did not. Now $u_{1}, u_{2}, U_{3}, w_{1}, w_{2}, \ldots, w_{6}$ are out of play, $z\left(G_{n, k+12}\right)=z\left(G_{n, k}\right)-8=8 m, P\left(G_{n, k+12}\right)=\left\{w_{8}\right\}, T\left(G_{n, k+12}\right)=\left\{w_{7}\right\}$, and $\left(w_{7}, w_{8}\right) \in F\left(G_{n, k+12}\right)$. Thus $G_{n, k+12} \in \mathcal{L}_{2}(8 m)(a)$ and $\mathcal{A}$ wins by Lemma 5.4.

- If $\mathcal{B}$ chooses one of $\left(u_{3}, w_{3}\right)$ or $\left(w_{1}, w_{2}\right)$, then $\mathcal{A}$ chooses the other edge.

If $\mathcal{B}$ chooses $\left(w_{1}, w_{3}\right)$, then $\mathcal{A}$ chooses $\left(u_{3}, w_{3}\right)$.

In each case, the resulting graph satisfies:

1. $(z(G), p(G), t(G))=(8 m+4,1,2)$,

2. if $P(G)=\left\{x_{1}\right\}$ and $T(G)=\left\{x_{2}, x_{3}\right\}$, then $\left(x_{2}, x_{3}\right) \in E(G)$, and

3. all other pairs are in $(E(G) \cup F(G))^{c}$.

Gameplay is identical in each case, so we may consider the first case. $\mathcal{B}$ has three choices: $\left(w_{1}, w_{3}\right),\left(w_{1}, w_{4}\right)$, or $\left(w_{3}, w_{4}\right)$.

- If $\mathcal{B}$ chooses $\left(w_{1}, w_{3}\right)$ then $\mathcal{A}$ chooses $\left(w_{2}, w_{4}\right)$. 
- If $\mathcal{B}$ chooses $\left(w_{1}, w_{4}\right)$ then $\mathcal{A}$ chooses $\left(w_{2}, w_{3}\right)$.

- If $\mathcal{B}$ chooses $\left(w_{3}, w_{4}\right)$ then $\mathcal{A}$ chooses $\left(w_{1}, w_{3}\right)$.

In each case, the resulting graph is in $\mathcal{L}_{1}(8 m+4)(b)$ and gameplay is identical, so without loss of generality we may consider the first case. $\mathcal{B}$ again has three choices: $\left(w_{3}, w_{4}\right),\left(w_{3}, w_{5}\right)$, or $\left(w_{4}, w_{5}\right)$.

- If $\mathcal{B}$ chooses $\left(w_{3}, w_{4}\right)$ then $\mathcal{A}$ chooses $\left(w_{4}, w_{5}\right) . \quad \mathcal{B}$ must choose $\left(w_{5}, w_{6}\right)$. $\mathcal{A}$ chooses $\left(w_{5}, w_{7}\right)$. $\mathcal{B}$ must choose $\left(w_{6}, w_{8}\right)$. $\mathcal{A}$ chooses $\left(w_{7}, w_{8}\right)$. Now $u_{1}, u_{2}, U_{3}, w_{1}, w_{2}, \ldots, w_{5}$ are out of play, $z\left(G_{n, k+12}\right)=z\left(G_{n, k}\right)-8=8 m$, $p\left(G_{n, k+12}\right)=0, T\left(G_{n, k+12}\right)=\left\{w_{6}, w_{7}, w_{8}\right\}$, and $\left(w_{6}, w_{8}\right),\left(w_{7}, w_{8}\right) \in E_{k+12}$. Thus $G_{n, k+12} \in \mathcal{L}_{3}(8 m)(b)$ and $\mathcal{A}$ wins by induction.

- If $\mathcal{B}$ chooses one of $\left(w_{3}, w_{5}\right)$ or $\left(w_{4}, w_{5}\right)$, then $\mathcal{A}$ chooses the other edge. $\mathcal{B}$ must choose $\left(w_{4}, w_{6}\right)$. $\mathcal{A}$ chooses $\left(w_{5}, w_{7}\right)$. $\mathcal{B}$ has two choices: $\left(w_{6}, w_{7}\right)$ or $\left(w_{6}, w_{8}\right)$. $\mathcal{A}$ chooses whichever edge $\mathcal{B}$ did not. Now $u_{1}, u_{2}, U_{3}, w_{1}, w_{2}, \ldots, w_{6}$ are out of play, $z\left(G_{n, k+12}\right)=z\left(G_{n, k}\right)-8=8 m, P\left(G_{n, k+12}\right)=\left\{w_{8}\right\}, T\left(G_{n, k+12}\right)=\left\{w_{7}\right\}$, and $\left(w_{7}, w_{8}\right) \in F\left(G_{n, k+12}\right)$. Thus $G_{n, k+12} \in \mathcal{L}_{2}(8 m)(a)$ and $\mathcal{A}$ wins by Lemma 5.4 .

Theorem 5.6. For $n \geq 12, f_{\{B 3, T, C\}}(n)=\mathcal{B} \Longleftrightarrow n \equiv 1,2(\bmod 4)$.

Proof. For small values of $n$, an exhaustive case analysis can be carried out by hand calculation.

\begin{tabular}{c|c|c|c|c|c|c|c|c|c}
$n$ & 3 & 4 & 5 & 6 & 7 & 8 & 9 & 10 & 11 \\
\hline$f_{\{B 3, T, C\}}(n)$ & $\mathcal{B}$ & $\mathcal{A}$ & $\mathcal{B}$ & $\mathcal{A}$ & $\mathcal{B}$ & $\mathcal{A}$ & $\mathcal{A}$ & $\mathcal{B}$ & $\mathcal{B}$
\end{tabular}

For larger values of $n$, we will prove a statement that is equivalent to the theorem: For $\mathrm{n} \geq 12, f_{\{B 3, T, C\}}(n)=\mathcal{B} \Longleftrightarrow n \equiv 1,2,5,6(\bmod 8)$.

$n \equiv 0(\bmod 8)$ :

Let $n=8 m$ for some $m \geq 2$. By Lemma 5.1, $\mathcal{A}$ can choose edges $e_{1}, e_{3}, \ldots, e_{21}$ so that $G_{n, 21} \in \mathcal{L}_{1}(n-15)$. Then by Lemma $5.3, \mathcal{A}$ can choose the edge $e_{23}$ so that $G_{n, 23} \in\left(\mathcal{L}_{2}(n-16) \cup \mathcal{L}_{3}(n-16)\right)$. Since $n-16 \equiv 0(\bmod 8)$, by Lemmas 5.4 and $5.5, \mathcal{A}$ wins.

$n \equiv 1(\bmod 8):$

Let $n=8 m+1$ for some $m \geq 2$. By Lemma $5.2, \mathcal{B}$ can choose edges $e_{2}, e_{4}, \ldots, e_{18}$ so that $G_{n, 18} \in \mathcal{L}_{1}(n-13)$. Then by Lemma $5.3, \mathcal{B}$ can choose the edges $e_{20}, e_{22}, e_{24}$ so that $G_{n, 24} \in\left(\mathcal{L}_{2}(n-17) \cup \mathcal{L}_{3}(n-17)\right)$. Since $n-17 \equiv 0(\bmod 8)$, by Lemmas 5.4 and $5.5, \mathcal{B}$ wins.

$n \equiv 2(\bmod 8):$

Let $n=8 m+2$ for some $m \geq 1$. By Lemma $5.2, \mathcal{B}$ can choose edges $e_{2}, e_{4}, \ldots, e_{12}$ so that $G_{n, 9} \in \mathcal{L}_{1}(n-9)$. Then by Lemma $5.3, \mathcal{B}$ can choose the edge $e_{14}$ so that 
$G_{n, 14} \in\left(\mathcal{L}_{2}(n-10) \cup \mathcal{L}_{3}(n-10)\right)$. Since $n-10 \equiv 0(\bmod 8)$, by Lemmas 5.4 and $5.5, \mathcal{B}$ wins.

\section{$n \equiv 3(\bmod 8):$}

Let $n=8 m+3$ for some $m \geq 2$. By Lemma $5.1, \mathcal{A}$ can choose edges $e_{1}, e_{3}, \ldots, e_{21}$ so that $G_{n, 21} \in \mathcal{L}_{1}(n-15)$. Then by Lemma $5.3, \mathcal{A}$ can choose the edges $e_{23}, e_{25}, e_{27}$ so that $G_{n, 27} \in\left(\mathcal{L}_{2}(n-19) \cup \mathcal{L}_{3}(n-19)\right)$. Since $n-19 \equiv 0(\bmod 8)$, by Lemmas 5.4 and $5.5, \mathcal{A}$ wins.

\section{$n \equiv 4(\bmod 8):$}

Let $n=8 m+4$ for some $m \geq 1$. By Lemma $5.1, \mathcal{A}$ can choose edges $e_{1}, e_{3}, \ldots, e_{15}$ so that $G_{n, 15} \in \mathcal{L}_{1}(n-11)$. Then by Lemma $5.3, \mathcal{A}$ can choose the edge $e_{17}$ so that $G_{n, 17} \in\left(\mathcal{L}_{2}(n-12) \cup \mathcal{L}_{3}(n-12)\right)$. Since $n-12 \equiv 0(\bmod 8)$, by Lemmas 5.4 and $5.5, \mathcal{A}$ wins.

\section{$n \equiv 5(\bmod 8):$}

Let $n=8 m+5$ for some $m \geq 1$. By Lemma $5.2, \mathcal{B}$ can choose edges $e_{2}, e_{4}, \ldots, e_{12}$ so that $G_{n, 12} \in \mathcal{L}_{1}(n-9)$. Then by Lemma $5.3, \mathcal{B}$ can choose the edges $e_{14}, e_{16}, e_{18}$ so that $G_{n, 18} \in\left(\mathcal{L}_{2}(n-13) \cup \mathcal{L}_{3}(n-13)\right)$. Since $n-13 \equiv 0(\bmod 8)$, by Lemmas 5.4 and $5.5, \mathcal{B}$ wins.

$n \equiv 6(\bmod 8):$

Let $n=8 m+6$ for some $m \geq 1$. By Lemma $5.2, \mathcal{B}$ can choose edges $e_{2}, e_{4}, \ldots, e_{18}$ so that $G_{n, 18} \in \mathcal{L}_{1}(n-13)$. Then by Lemma $5.3, \mathcal{B}$ can choose the edge $e_{20}$ so that $G_{n, 20} \in\left(\mathcal{L}_{2}(n-14) \cup \mathcal{L}_{3}(n-14)\right)$. Since $n-14 \equiv 0(\bmod 8)$, by Lemmas 5.4 and $5.5, \mathcal{B}$ wins.

\section{$n \equiv 7(\bmod 8):$}

Let $n=8 m+7$ for some $m \geq 1$. By Lemma $5.1, \mathcal{A}$ can choose edges $e_{1}, e_{3}, \ldots, e_{15}$ so that $G_{n, 15} \in \mathcal{L}_{1}(n-11)$. Then by Lemma $5.3, \mathcal{A}$ can choose the edges $e_{17}, e_{19}, e_{21}$ so that $G_{n, 21} \in\left(\mathcal{L}_{2}(n-15) \cup \mathcal{L}_{3}(n-15)\right)$. Since $n-15 \equiv 0(\bmod 8)$, by Lemmas 5.4 and $5.5, \mathcal{A}$ wins.

\section{References}

[1] S.C. Cater, F. Harary, and R.W. Robinson, One-color triangle avoidance games, Congressus Numerantium 153 (2001), 211-221.

[2] P. Gordinowicz and P. Pralat, The first player wins the one-colour triangle avoidance game on 16 vertices, Discussiones Mathematicae Graph Theory, to appear.

[3] F. Harary, Achievement and avoidance games for graphs, Annals of Discrete Mathematics 13 (1982), 111-119.

[4] N. Mehta, Graph Games, Ph.D. thesis, Ohio State University, 2010.

[5] N. Mehta, Á. Seress, Bounded Degree, Triangle Avoidance Graph Games, submitted.

[6] P. Pralat, A note on the one-colour avoidance game on graphs, Journal of Combinatorial Mathematics and Combinatorial Computing 75 (2010), 85-94.

[7] Á. Seress, On Hajnal's triangle-free game, Graphs and Combinatorics 8 (1992), 75-79. 